\title{
On the Effect of Surface Heat-Flux Heterogeneities on the Mixed-Layer-Top Entrainment
}

\author{
Matthias Sühring • Björn Maronga - Florian Herbort • \\ Siegfried Raasch
}

Received: 31 July 2013 / Accepted: 4 February 2014 / Published online: 13 March 2014

(C) The Author(s) 2014. This article is published with open access at Springerlink.com

\begin{abstract}
We used a set of large-eddy simulations to investigate the effect of one-dimensional stripe-like surface heat-flux heterogeneities on mixed-layer top entrainment. The profiles of sensible heat flux and the temporal evolution of the boundary-layer depth revealed decreased entrainment for small heat-flux amplitudes and increased entrainment for large heat-flux amplitudes, compared to the homogeneously-heated mixed layer. For large heat-flux amplitudes the largest entrainment was observed for patch sizes in the order of the boundary-layer depth, while for significantly smaller or larger patch sizes entrainment was similar as in the homogeneous case. In order to understand the underlying physics of this impact, a new approach was developed to infer local information on entrainment by means of the local flux divergence. We found an entrainment maximum over the centre of the stronger heated surface patch, where thermal energy is accumulated by the secondary circulation (SC) that was induced by the surface heterogeneity. Furthermore, we observed an entrainment maximum over the less heated patch as well, which we suppose is to be linked to the SC-induced horizontal flow convergence at the top of the convective boundary layer (CBL). For small heat-flux amplitudes a counteracting effect dominates that decreases entrainment, which we suppose is the horizontal advection of cold air in the lower, and warm air in the upper, CBL by the SC, stabilizing the CBL and thus weakening thermal convection. Moreover, we found that a mean wind can reduce the heterogeneity-induced impact on entrainment. If the flow is aligned perpendicular to the border between the differentially-heated patches, the SC and thus its impact on entrainment vanishes due to increased horizontal mixing, even for moderate wind speeds. However, if the flow is directed parallel to the border between the differentially-heated patches, the SC and thus its impact on entrainment persists.
\end{abstract}

Keywords Convective boundary layer · Encroachment · Entrainment · Large-eddy simulation · Surface heterogeneity

M. Sühring $(\bowtie) \cdot$ B. Maronga $\cdot$ F. Herbort $\cdot$ S. Raasch

Institut für Meteorologie und Klimatologie, Leibniz Universität Hannover,

Herrenhäuser Str. 2, 30419 Hannover, Germany

e-mail: suehring@muk.uni-hannover.de 


\section{Introduction}

Entrainment at the top of the convective boundary layer (CBL) describes the mixing of nonturbulent, warm and dry tropospheric air into the turbulent CBL through a stably-stratified interfacial layer (e.g. Sullivan et al. 1998). It is an important process that affects the development of the daytime CBL, because it regulates the exchange of sensible and latent heat, momentum (Conzemius and Fedorovich 2006), as well as chemical species (Ouwersloot et al. 2011) between the CBL and the free atmosphere. The underlying physics are on much smaller scales than the grid size used in numerical weather prediction (NWP) models, hence NWP models rely on accurate parametrizations of entrainment to incorporate its effect on the CBL evolution.

When neglecting large-scale synoptic forcing, the CBL grows only by surface heating and by entrainment. The CBL growth due to pure thermodynamic heating at the surface (assuming an incompressible fluid without thermal expansion) is termed encroachment (Stull 1988), which explains about 70-90\% of the CBL growth (Stull 1976; Canut et al. 2010). The remaining 10-30\% can be ascribed to the entrainment of warm air. The physics and magnitude of entrainment depend strongly on the surface forcing and on the structure of the interfacial layer between the CBL and the troposphere, i.e. on its thickness and strength, which has been investigated in numerous studies using remote sensing techniques (e.g. Angevine et al. 1994; Davis et al. 1997, 2000; Beyrich and Gryning 1998; Angevine 1999; Grabon et al. 2010; Träumner et al. 2011), aircraft observations (e.g. Lenschow et al. 1999; Bange et al. 2007; Canut et al. 2010) and large-eddy simulations (LES) (e.g. Sullivan et al. 1998; Otte and Wyngaard 2001; Fedorovich et al. 2004; Brooks and Fowler 2012). Furthermore, entrainment physics depend also on the presence of elevated wind shear in the inversion layer that is accompanied by an increase in the magnitude of entrainment (Davis et al. 1997; Kim et al. 2003; Pino et al. 2003; Conzemius and Fedorovich 2006; Canut et al. 2010). The same applies also to the presence of roll convection and gravity waves (Lothon et al. 2009). Several LES studies reported also an effect of heterogeneous surface heating on entrainment (Avissar and Schmidt 1998; Raasch and Harbusch 2001; Patton et al. 2005; van Heerwaarden and de Arellano 2008; Ouwersloot et al. 2011; Maronga and Raasch 2013), though this and the effect on the structure of the interfacial layer are still not well understood to date.

The local impact of a heterogeneous forcing on the structure of the interfacial layer was studied by aircraft observations (Kang et al. 2007) and LES (van Heerwaarden and de Arellano 2008; Fesquet et al. 2009; Wang et al. 2011; Maronga and Raasch 2013). All of these studies reported a correlation between the local CBL depth $z_{\mathrm{i}}$ and the underlying surface sensible heat flux, where larger values of $z_{\mathrm{i}}$ were observed over stronger heated surface patches and vice versa. In their LES study over one-dimensional stripe-like surface patches van Heerwaarden and de Arellano (2008), hereafter HA08, attributed these spatial variations in the local $z_{\mathrm{i}}$ to rising secondary circulation (SC) updrafts that led to increased entrainment over the stronger heated patch. Maronga and Raasch (2013) used LES to study the effect of irregularly distributed surface heterogeneity on the CBL during the LITFASS-2003 experiment (Beyrich and Mengelkamp 2006). In their study the local $z_{\mathrm{i}}$ was highly correlated with the underlying surface heterogeneity pattern, whereas the SCs were found to be located at the edges of the surface patches and did not extend to the centre of the surface patches. Increased entrainment associated with SC updrafts thus did not explain the spatial variations in the local $z_{\mathrm{i}}$ over the LITFASS terrain, so that Maronga and Raasch (2013) attributed the spatial variations in the local $z_{i}$ to spatial variations in encroachment. Based on LES for the LITFASS-2003 experiment, Sühring and Raasch (2013) conducted a correlation analysis 
between the turbulent heat-flux patterns in the CBL and the underlying surface heat-flux pattern. They showed that signals from the surface heterogeneity extend throughout the entire CBL, resulting in increased entrainment over stronger heated patches and decreased entrainment over less heated patches. However, as local profiles of the sensible heat flux revealed spatially varying heating of the CBL, indicating spatially varying encroachment, it was not possible in their study to separate entrainment and encroachment, so that the spatial variations in the local $z_{\mathrm{i}}$ can be due to spatial variations in both entrainment and encroachment.

Henceforth, for a proper distinction, the entrainment of air from the free atmosphere into the CBL at a certain location (i.e. a finite column of air) is hereafter referred to as local entrainment, while entrainment derived from horizontal mean values over a horizontally extended domain is referred to as domain-averaged entrainment.

Even though various studies investigated the effect of surface heterogeneities on domainaveraged entrainment, it is still an open question whether a heterogeneous surface forcing affects domain-averaged entrainment, and if so, whether it is increased or decreased. One reason might be attributed to the use of different methods to define the magnitude of domainaveraged entrainment over heterogeneous terrain. Previous studies used either the entrainment velocity $w_{\mathrm{e}}=\mathrm{d} z_{\mathrm{i}} / \mathrm{d} t$, which is the temporal derivative of $z_{\mathrm{i}}$, or the profile of sensible heat flux at the top of the CBL, particularly its minimum value and the region with negative flux, which are common entrainment measures (e.g. van Zanten et al. (1999)). However, horizontally-averaged heat-flux profiles in the entrainment layer obtained from LES should be interpreted very carefully with respect to the magnitude of entrainment, because local entrainment events at different heights can be smoothed out by the horizontal averaging (Lilly 2002). This is even more critical over heterogeneously-heated surfaces where the vertical level of the entrainment layer varies spatially due to the spatial variations of $z_{\mathrm{i}}$. This results in a deeper region with negative heat flux for the heterogeneously-heated CBL compared to the homogeneously-heated CBL (HA08), suggesting different magnitudes of entrainment just by horizontal averaging.

Maronga and Raasch (2013) reported slightly decreased domain-averaged entrainment over the heterogeneously-heated LITFASS terrain compared to a homogeneous reference simulation, indicated by heat-flux profiles and $w_{\mathrm{e}}$. They supposed that the existing SCs partly redirect the vertical transport to the horizontal transport, which in turn decreases entrainment. In contrast, based on profiles of sensible heat flux, Avissar and Schmidt (1998), and Raasch and Harbusch (2001) found increased entrainment in their LES over idealized sensible surface heat-flux heterogeneities compared to homogeneous runs. Moreover, Avissar and Schmidt (1998) observed increased entrainment for increasing patch size. Patton et al. (2005) investigated the impact of idealized surface heterogeneities on CBL structure and found only small changes in $w_{\mathrm{e}}$ for varying heterogeneity scales, where $w_{\mathrm{e}}$ slightly decreased for patch sizes in the order of $z_{\mathrm{i}}$, but slightly increased for significantly larger patch sizes. However, in contrast to Avissar and Schmidt (1998) and Raasch and Harbusch (2001) the considered surface heat-flux heterogeneities were less pronounced in amplitude, indicating that the heterogeneity effect on entrainment was comparatively small in their study. HA08 observed, by means of vertical heat-flux profiles, increased entrainment for cases with large heterogeneity amplitudes and decreased entrainment for cases with small heterogeneity amplitudes, compared to a homogeneous reference case. However, they discarded this finding and attributed these modifications to the effect of the horizontal averaging, because they found the temporal evolution of $z_{\mathrm{i}}$ not affected by the heterogeneous surface heating. Based on LES over one-dimensional stripe-like surface patches, Ouwersloot et al. (2011), hereafter O11, pointed out that the effect of surface heterogeneities on entrainment is diverse, even though their results are based on differences in $z_{\mathrm{i}}$ at a certain point in time only, which 
could also result from differences in the model spin-up (HA08). While they found increased entrainment for large heterogeneity amplitudes compared to a homogeneously-heated CBL, they observed decreased entrainment for small heterogeneity amplitudes. They attributed these findings to the existing SC, where the uprising branch of the SC allows the thermals to penetrate the inversion more rigorously. At the same time the subsiding branch of the SC suppresses the thermal convection. Depending on the strength of the SC, either the promoting or the suppressive effect of the SC on entrainment dominates, which in turn indicates that the suppressive effect is saturated at some point. However, the underlying physics are still unknown and a detailed investigation is still pending. Moreover, O11 showed that the largest impact on entrainment occurs when the patch size is in the order of $z_{\mathrm{i}}$. For significantly smaller or larger patches no effect was observed, because the SC did not reach the top of the CBL or was weak and thus of minor importance for the entrainment. Even though the heterogeneity amplitudes are not directly comparable, this finding is in contrast to Avissar and Schmidt (1998) who observed increased entrainment for increasing patch size.

These different findings in the literature suggest that the effect of surface heterogeneity on entrainment depends in a complex manner on the atmospheric conditions and the type of surface heterogeneity, stressing the need for a comprehensive and systematic investigation of whether and how a heterogeneous surface forcing affects entrainment. In order to focus independently on effects induced by different heterogeneity amplitude and scale, which would be impossible for realistic surface heterogeneity where various heterogeneity amplitudes and scales are superimposed, we prescribe idealized surface heterogeneities. In a first step, based on a series of LES runs over one-dimensional stripe-like surface patches, we analyze how the heterogeneous forcing and the existing SC modify the CBL structure. To quantify domain-averaged entrainment over heterogeneous terrain former studies used either the profiles of sensible heat flux or the temporal evolution of $z_{\mathrm{i}}$, while local information on entrainment was often inferred from local values of $z_{i}$. However, heat-flux profiles might be affected by horizontal averaging, $z_{\mathrm{i}}$ might be affected by differences in the model spin-up for different surface heterogeneities, and local values of $z_{\mathrm{i}}$ incorporate also the effect of encroachment, making it difficult to separate the heterogeneity effect on entrainment. In order to divide the boundary-layer growth into an entrainment portion and into an encroachment portion, we hence develop a new method based on the local flux divergence. In this way we provide local estimates of the entrainment rate, so that the method is also independent on horizontal averaging. Based on this approach, we investigate how the heterogeneity-induced SC affects local entrainment, and how the identified possible responsible processes interact with each other depending on the heterogeneous forcing. Subsequently, in order to achieve a more comprehensive view of the heterogeneityinduced impact on entrainment we perform a series of LES runs with a broader range of heterogeneity amplitudes and patch sizes, as well as different wind speeds and wind directions.

The paper is organized as follows: Sect. 2 describes the LES model, the simulation set-ups and the heterogeneous surface forcing, as well as the statistical analysis methods. Section 3 gives a detailed analysis of the structure of the heterogeneously-heated CBL. Subsequently, the method to determine the local entrainment rate is introduced and the results of this analysis are presented. Moreover, the possible heterogeneity-induced mechanisms modifying entrainment are identified and discussed. At the end of Sect. 3 a more comprehensive picture of the effect of varying heat-flux amplitude, patch size and mean wind on entrainment is presented. Section 4 gives a summary. 


\section{Methods}

\subsection{LES Model and Numerical Experiments}

The PArallelized LES Model PALM (Raasch and Etling 1998; Raasch and Schröter 2001), revision $993,{ }^{1}$ was used for the numerical simulations, and solves the non-hydrostatic incompressible Boussinesq equations. For the subgrid model the kinetic energy scheme of Deardorff (1980) was used. The advection terms were discretized by a fifth-order scheme (Wicker and Skamarock 2002), and for the time integration a third-order Runge-Kutta scheme (Williamson 1980) was used. Cyclic lateral boundary conditions were used, and between the surface and the first grid level Monin-Obukhov similarity theory was applied.

In the present study we focused on the dry CBL. All numerical experiments were initialized by a potential temperature profile $\theta(z)$ where $\theta$ was constant up to $800 \mathrm{~m}$. This layer was capped by an inversion with a temperature jump of $2 \mathrm{~K}$, and the above-lying free atmosphere had a lapse rate of $0.006 \mathrm{~K} \mathrm{~m}^{-1}$. At the beginning of each simulation randomly distributed perturbations were imposed on the horizontal velocity fields to initiate turbulence. The surface heterogeneity in our simulations was prescribed by a horizontally varying surface sensible heat flux. A homogeneous roughness length of $z_{0}=0.1 \mathrm{~m}$ was used.

In order to create heterogeneous forcing, we divided the model surface into alternating stronger and less heated patches along the $x$-axis. The surface sensible heat flux $H_{0}(x)$ on the respective patch deviates from the domain-averaged surface heat flux by a certain percentage, described by the parameter $A \in[0,1]$. The domain-averaged surface heat flux was constant with a value of $0.1 \mathrm{~K} \mathrm{~m} \mathrm{~s}^{-1}$ in all performed simulations. In order to avoid different spin-up times for turbulence over different patches at the beginning of the simulation, the surface was heated homogeneously during the first hour of the simulation.

In a first step we varied the amplitude of the surface heterogeneity by varying $A$ between 0 (the homogeneous reference case) and 1 with increments of 0.1 for a constant patch size $L=3,200 \mathrm{~m}$. In a second step we varied $L$ for given $A=0.5$, where $L$ ranged from values significantly smaller to significantly larger than $z_{\mathrm{i}}$. An overview of all performed simulations is given in Table 1. The name of the particular simulation is derived from the parameter $A$ and $L$, for example, A50L32 is a simulation with $L=3,200 \mathrm{~m}$ and $A=0.5$, where $H_{0}(x)$ is $50 \%$ larger (smaller) over the stronger (less) heated patch compared to the horizontal mean value.

Raasch and Harbusch (2001) showed that a mean wind can modify the impact of surface heat-flux heterogeneities on the CBL. Depending on the wind direction with respect to the surface heterogeneity the impact can be reduced, even for moderate wind speeds. In order to study the relative importance of surface heterogeneities on entrainment under different wind regimes, we varied the geostrophic wind speed and direction for given $A=0.5$ and $L=3200 \mathrm{~m}$. The geostrophic wind was adjusted in such a way that the mean boundary-layer flow is directed parallel or perpendicular to the heterogeneous $x$-axis during the simulation. To separate the heterogeneity effect from the effect of varying wind shear on entrainment, we performed homogeneous reference simulations with identical geostrophic forcing. The name of the particular simulation with mean wind (see Table 1) was derived from the wind direction and from the wind speed, where $U$ and $V$ denote simulations with mean CBL wind perpendicular and parallel to the heterogeneity, respectively.

For the studies with varying mean wind speed and heterogeneity amplitude and scale our model domain was $6.4 \mathrm{~km} \times 25.6 \mathrm{~km} \times 3.5 \mathrm{~km}$ in the $x$-, $y$-, and $z$-directions, respectively. For patch sizes larger than $L=3,200 \mathrm{~m}$ we extended the domain in the $x$-direction so that

1 The code can be accessed under http://palm.muk.uni-hannover.de/browser/?rev=993. 
Table 1 Parameters of the performed simulations

\begin{tabular}{|c|c|c|c|c|c|c|}
\hline Name & $\begin{array}{l}\text { Grid } \\
x \times y \times z\end{array}$ & $\begin{array}{l}A \\
-\end{array}$ & $\begin{array}{l}\Delta H_{0} \\
\left(\mathrm{~K} \mathrm{~m} \mathrm{~s}^{-1}\right)\end{array}$ & $\begin{array}{l}L \\
(\mathrm{~m})\end{array}$ & $\begin{array}{l}U_{\mathrm{CBL}} \\
\left(\mathrm{m} \mathrm{s}^{-1}\right)\end{array}$ & $\begin{array}{l}V_{\mathrm{CBL}} \\
\left(\mathrm{m} \mathrm{s}^{-1}\right)\end{array}$ \\
\hline HOM & $512 \times 2048 \times 192$ & 0.0 & 0.0 & - & 0.0 & 0.0 \\
\hline HOM* & $512 \times 32000 \times 192$ & 0.0 & 0.0 & - & 0.0 & 0.0 \\
\hline \multicolumn{7}{|l|}{ Amplitude } \\
\hline A10L32 & $512 \times 2048 \times 192$ & 0.1 & 0.02 & 3200 & 0.0 & 0.0 \\
\hline A20L32 & $512 \times 2048 \times 192$ & 0.2 & 0.04 & 3200 & 0.0 & 0.0 \\
\hline A20L32* & $512 \times 32000 \times 192$ & 0.2 & 0.04 & 3200 & 0.0 & 0.0 \\
\hline A30L32 & $512 \times 2048 \times 192$ & 0.3 & 0.06 & 3200 & 0.0 & 0.0 \\
\hline A40L32 & $512 \times 2048 \times 192$ & 0.4 & 0.08 & 3200 & 0.0 & 0.0 \\
\hline A50L32 & $512 \times 2048 \times 192$ & 0.5 & 0.1 & 3200 & 0.0 & 0.0 \\
\hline A50L32* & $512 \times 32000 \times 192$ & 0.5 & 0.1 & 3200 & 0.0 & 0.0 \\
\hline A60L32 & $512 \times 2048 \times 192$ & 0.6 & 0.12 & 3200 & 0.0 & 0.0 \\
\hline A70L32 & $512 \times 2048 \times 192$ & 0.7 & 0.14 & 3200 & 0.0 & 0.0 \\
\hline A80L32 & $512 \times 2048 \times 192$ & 0.8 & 0.16 & 3200 & 0.0 & 0.0 \\
\hline A90L32 & $512 \times 2048 \times 192$ & 0.9 & 0.18 & 3200 & 0.0 & 0.0 \\
\hline A100L32 & $512 \times 2048 \times 192$ & 1.0 & 0.2 & 3200 & 0.0 & 0.0 \\
\hline \multicolumn{7}{|l|}{ Patch size } \\
\hline A50L01 & $512 \times 2048 \times 192$ & 0.5 & 0.1 & 100 & 0.0 & 0.0 \\
\hline A50L02 & $512 \times 2048 \times 192$ & 0.5 & 0.1 & 200 & 0.0 & 0.0 \\
\hline A50L04 & $512 \times 2048 \times 192$ & 0.5 & 0.1 & 400 & 0.0 & 0.0 \\
\hline A50L08 & $512 \times 2048 \times 192$ & 0.5 & 0.1 & 800 & 0.0 & 0.0 \\
\hline A50L08* & $512 \times 32000 \times 192$ & 0.5 & 0.1 & 800 & 0.0 & 0.0 \\
\hline A50L16 & $512 \times 2048 \times 192$ & 0.5 & 0.1 & 1600 & 0.0 & 0.0 \\
\hline A50L16* & $512 \times 32000 \times 192$ & 0.5 & 0.1 & 1600 & 0.0 & 0.0 \\
\hline A50L64 & $1024 \times 2048 \times 192$ & 0.5 & 0.1 & 6400 & 0.0 & 0.0 \\
\hline A50L96 & $1536 \times 2048 \times 192$ & 0.5 & 0.1 & 9600 & 0.0 & 0.0 \\
\hline \multicolumn{7}{|l|}{ Wind } \\
\hline U06 & $512 \times 2048 \times 192$ & 0.5 & 0.1 & 3200 & 0.6 & 0.0 \\
\hline U12 & $512 \times 2048 \times 192$ & 0.5 & 0.1 & 3200 & 1.2 & 0.0 \\
\hline $\mathrm{U} 25$ & $512 \times 2048 \times 192$ & 0.5 & 0.1 & 3200 & 2.5 & 0.0 \\
\hline U50 & $512 \times 2048 \times 192$ & 0.5 & 0.1 & 3200 & 5.0 & 0.0 \\
\hline V06 & $512 \times 2048 \times 192$ & 0.5 & 0.1 & 3200 & 0.0 & 0.6 \\
\hline V12 & $512 \times 2048 \times 192$ & 0.5 & 0.1 & 3200 & 0.0 & 1.2 \\
\hline V25 & $512 \times 2048 \times 192$ & 0.5 & 0.1 & 3200 & 0.0 & 2.5 \\
\hline V50 & $512 \times 2048 \times 192$ & 0.5 & 0.1 & 3200 & 0.0 & 5.0 \\
\hline
\end{tabular}

The number of grid points is shown in the $x-, y$-, and $z$-directions. $\Delta H_{0}$ is the amplitude of the surface heterogeneity determined by the parameter $A$, which describes the relative deviation of the surface sensible heat flux over the respective patch from the horizontal mean value; $L$ is the patch size; and $U_{\mathrm{CBL}}$ and $V_{\mathrm{CBL}}$ are the horizontal mean wind-speed components along and perpendicular the heterogeneous $x$-axis within the CBL during the simulation, respectively. Simulations marked with an asterisk are performed on larger domains

at least two differentially-heated patches fit into the model domain. We used an isotropic grid length of $12.5 \mathrm{~m}$ for all simulations. A sensitivity study (not shown) revealed this grid length to be sufficient, because the temporal evolution of $z_{\mathrm{i}}$, which is thought to be one of the 
most grid sensitive quantities (Sullivan and Patton 2011), did not change for decreasing grid spacing, indicating that the bulk of the entrainment processes was resolved in our simulations. Well above the entrainment layer the vertical grid was stretched to minimize computational resources, and a sponge layer prevented the reflection of gravity waves at the top of the model domain.

In Sect. 3.2 we present a method to determine local entrainment rates; this approach required an extended model domain in the $y$-direction to obtain a sufficiently large sample size. Therefore, we also performed simulations with a model domain of $6.4 \mathrm{~km} \times 400.0 \mathrm{~km} \times$ $3.5 \mathrm{~km}$ in the $x-, y$-, and $z$-directions for selected cases, which are marked by an asterisk. These enlarged simulations ran for $4 \mathrm{~h}$ of simulation time and entrainment rates were calculated during the last hour of the simulation. The simulations with the smaller domain ran for 1 $\mathrm{h}$ more, i.e. $5 \mathrm{~h}$, to highlight the different temporal evolution of the CBL over a longer period.

\subsection{Statistical Analysis}

The horizontal domain average of a quantity $\phi$ is indicated by squared brackets [], while the temporal average is indicated by an overbar. The surface forcing in our simulations is homogeneous along the $y$-axis. Hence, we can decompose a space and time-dependent variable into the spatial average along the $y$-axis and into a turbulent fluctuation as follows (e.g. Chen and Avissar 1994; Patton et al. 2005)

$$
\phi(x, y, z, t)=\langle\phi\rangle(x, z, t)+\phi^{\prime}(x, y, z, t),
$$

where $\langle\phi\rangle$ is the spatial average along the $y$-axis, hereafter referred to as the phase average, which can be further decomposed into a domain-averaged contribution $[\phi]$ and a mesoscale fluctuation $\widetilde{\phi} ; \phi^{\prime}$ is the turbulent fluctuation with respect to the phase average, and $t$ denotes the time. The phase average of a quantity is used to illustrate heterogeneity-induced differences in the CBL. We also applied phase averaging to obtain information on how the heterogeneous forcing affects the turbulent flux pattern. The resulting instantaneous phase-averaged vertical turbulent flux of $\phi$ is defined by

$$
\left\langle w^{\prime} \phi^{\prime}\right\rangle(x, z, t)=\langle(w(x, y, z, t)-\langle w\rangle(x, z, t))(\phi(x, y, z, t)-\langle\phi\rangle(x, z, t))\rangle,
$$

where $w$ is the vertical velocity. It should be noted that $\left\langle w^{\prime} \phi^{\prime}\right\rangle(x, z, t)$ is not representative of the total vertical transport at a given $x-z$ position, because it does not include the flux portion due to the $\mathrm{SC}$, hereafter referred to as the mesoscale flux, which is given by

$$
[\widetilde{w} \widetilde{\phi}](z, t)=[(\langle w\rangle(x, z, t)-[w](z, t))(\langle\phi\rangle(x, z, t)-[\phi](z, t))] .
$$

Particularly for cases with larger heat-flux amplitudes the mesoscale flux was found to exceed the turbulent flux in the middle of the CBL and in the entrainment layer (not shown), as already reported by HA08 for a similar heterogeneous forcing (see HA08, Fig. 6). Therefore, the phase-averaged turbulent flux does not necessarily provide information on the total transport at a certain location, so that the turbulent flux will not be used to infer information on local entrainment. Nevertheless, the turbulent flux patterns are used to provide a hint to the general total vertical transport patterns, because the mesoscale transport is linked to the turbulent transport over heterogeneous terrain where e.g. uprising thermals appear always at the same location (HA08).

A common measure of entrainment is $w_{\mathrm{e}}$ or the temporal evolution of the domain-averaged boundary-layer depth $\left[z_{\mathrm{i}}\right]$. Therefore, we calculated $\left[z_{\mathrm{i}}\right]$ by horizontal averaging the local boundary-layer depth $z_{\mathrm{i}}(x, y)$, according to the recent studies of HA08, O11, and Maronga and Raasch (2013). O11 evaluated different methods to determine $z_{\mathrm{i}}(x, y)$ and stated that 
the gradient method after Sullivan et al. (1998) gives the most reliable results. The gradient method defines $z_{\mathrm{i}}(x, y)$ as the height where the vertical gradient of the potential temperature $\partial \theta / \partial z$ is a maximum for given $x$ and $y$.

In Sect. 3.5 we discuss the effects of heterogeneity on the physics of entrainment. Therefore, we require local information on the turbulence intensity, which is usually provided by the turbulent kinetic energy (TKE). The turbulent fraction of a quantity is defined as the deviation from a reference value, which is usually the horizontal mean of the respective quantity in the LES. However, under horizontally heterogeneous conditions this method might fail, because the horizontal mean does not necessarily represent local mean conditions. Defining the TKE by means of the phase or temporal average is also not suitable as it would remove the mesoscale portion. This mesoscale portion contributes significantly to the entrainment flux, because entrainment events preferentially take place at the same location over heterogeneous terrain, as shown by HA08. We used the absolute value of the vorticity vector $\zeta_{\text {abs }}$ as a local measure of the turbulence intensity instead. The vorticity vector is given by

$$
\zeta_{i}=\epsilon_{i j k} \frac{\partial u_{j}}{\partial x_{k}}
$$

where $\epsilon_{i j k}$ is the Levi-Civita tensor and $u_{j}$ are the three velocity components; $\zeta_{\text {abs }}$ is based on local values and is not related to spatial or temporal averages such as the TKE. In contrast to the TKE, $\zeta_{\text {abs }}$ thus still includes local mesoscale fluctuations and is therefore better suited to study entrainment physics over heterogeneously-heated surfaces.

\section{Results}

\subsection{CBL Characteristics}

First of all we discuss here the impact of the heterogeneous surface forcing on the mean and local CBL structure for selected cases, i.e. A20L32*, A50L32*, A50L08*, A50L16* simulations, before we proceed to the discussion of the heterogeneity-induced effect on entrainment. Figure 1a shows the respective domain-averaged vertical profiles of $\theta$ after $4 \mathrm{~h}$ of simulated time. Simulation HOM* indicates a vertically well-mixed CBL, capped by an inversion with a potential temperature jump of about $2 \mathrm{~K}$. The A20L32* simulation exhibits a slightly cooler CBL with a slightly lower inversion height $\left(z_{\mathrm{i}}=1,010 \mathrm{~m}\right)$ compared to the $\mathrm{HOM}^{*}$ simulation, whereas the runs with larger heat-flux amplitude exhibit a warmer and deeper CBL (e.g. $z_{\mathrm{i}}=1,080 \mathrm{~m}$ for simulation A50L16*). Figure $1 \mathrm{~b}$ shows phase-averaged vertical profiles of $\theta$ over the centre of the stronger and less heated patches, hereafter referred to as the warm and cold patches, for the respective cases. The heterogeneous runs exhibit a temperature difference of about $0.5 \mathrm{~K}$ between the warm and cold patches. While the CBL is vertically well-mixed over the warm patches, except for the surface layer, it appears that the CBL over the cold patches is slightly stabilized and thus vertically less mixed, particularly in the upper part of the CBL. The inversion over the warm patches is higher than over the cold patches (about $50 \mathrm{~m}$ for simulation A50L32*), indicating that the CBL is not well-mixed horizontally, except for simulation A50L08* where the inversion height over the warm and cold patches is similar. Figure $2 \mathrm{a}-\mathrm{d}$ shows vertical cross-sections of the phase-averaged resolved-scale instantaneous turbulent vertical heat-flux pattern for the A20L32*, A50L32*, A50L08*, and A50L16* simulations, as well as thecorresponding SC indicated by the wind vectors. It should be noted that the $x$-axis is normalized with the respective patch size, meaning that the horizontal scales in Fig. 2 vary among the cases. Moreover, it should be noted that 

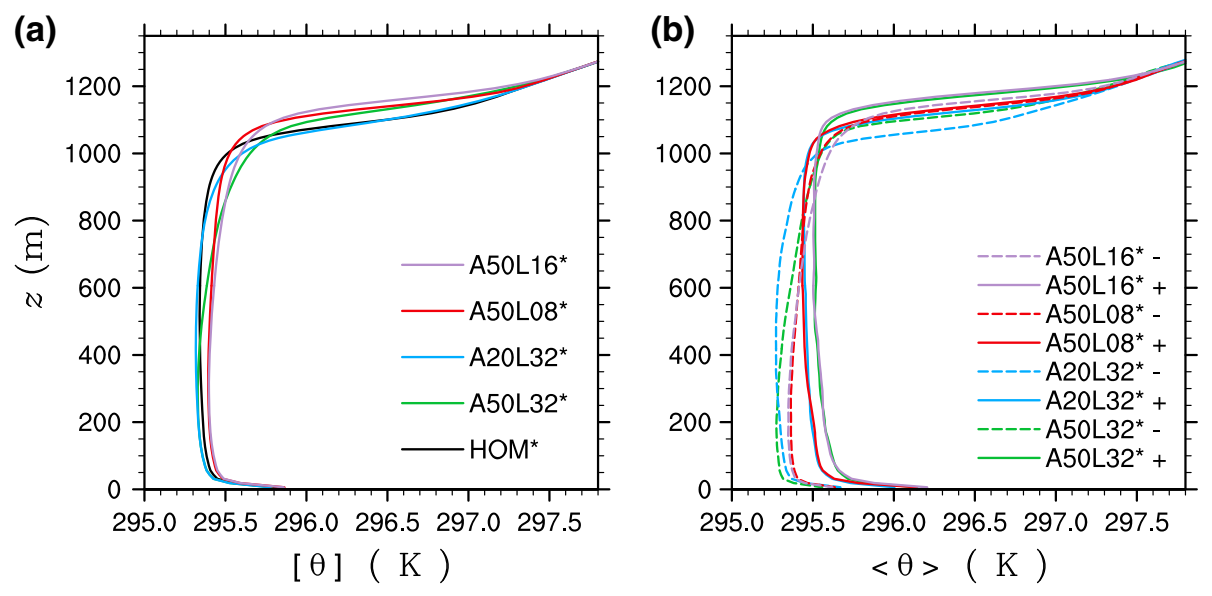

Fig. 1 Instantaneous vertical profiles of $\theta$ after $4 \mathrm{~h}$ of simulated time, a domain-averaged, b phase-averaged at the centre of the warm patches, indicated by plus, as well as at the centre of the cold patches, indicated by minus

(a)

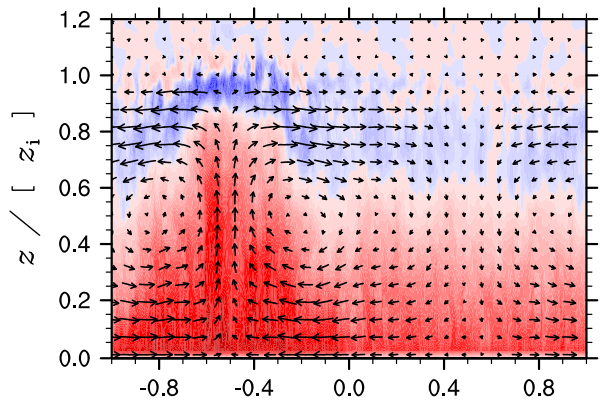

(c)

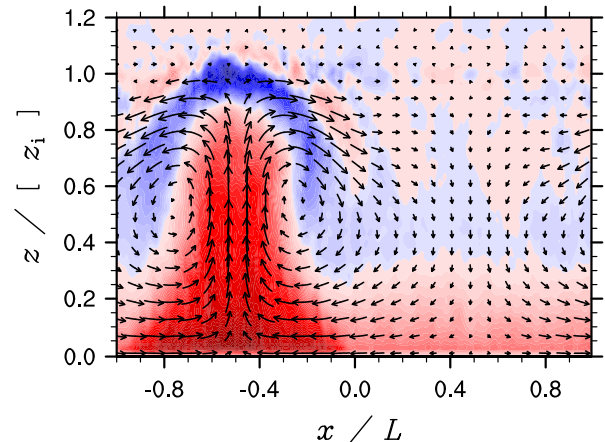

(b)

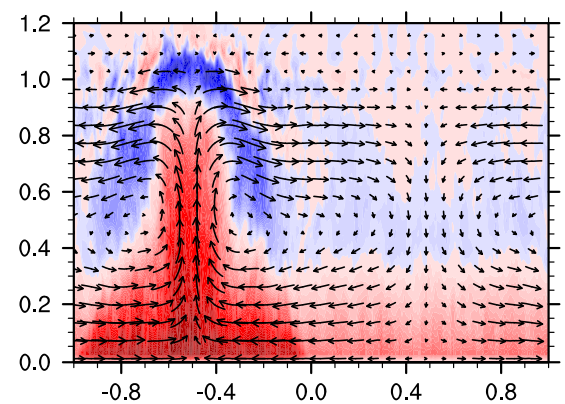

(d)

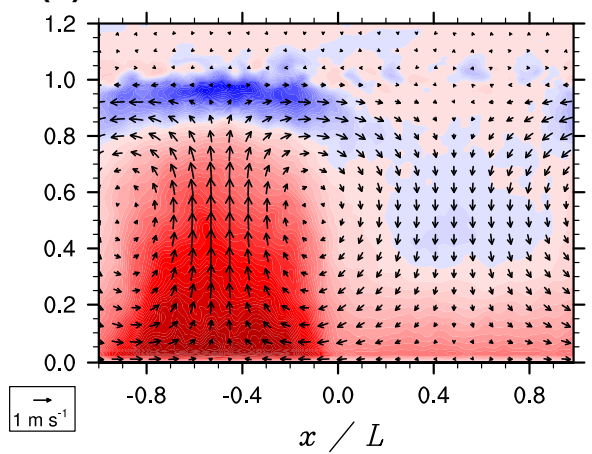

$\left(\stackrel{\mathrm{K}}{\mathrm{K} \mathrm{m} \mathrm{s}^{\prime} \mathrm{\theta}^{\prime}} \mathrm{s}^{-1}\right)^{\text {res }}$

$\begin{array}{lllllllll}-0.08 & -0.06 & -0.04 & -0.02 & -0.00 & 0.03 & 0.08 & 0.12 & 0.16\end{array}$

Fig. 2 Instantaneous vertical cross-section of the phase-averaged resolved-scale vertical turbulent sensible heat flux for the a A20L32*, b A50L32*, c A50L16*, and d A50L08* simulations after 4 h of simulated time. The corresponding phase-averaged flow is displayed by the wind vectors. The $x$-axis is normalized by the respective patch size; the $z$-axis is normalized by the respective domain-averaged CBL depth 
the displayed wind vectors do not represent the entire flow on the mesoscale. As the Coriolis force was considered, the horizontal branches of the SC turn to the right of the flow direction after some integration time, so that the SC is not a two-dimensional but a three-dimensional phenomenon. However, as the wind component in the $y$-direction was more than one order of magnitude smaller than in the $x$-direction, this effect of the Coriolis force is not considered further. The SCs vary in strength among the cases, indicated by the length of the wind vectors. The weakest SC can be observed for simulation A20L32*, while the strongest SCs can be observed for simulation A50L32* and A50L16*. For all cases the uprising branch of the SC is located over the centre of the warm patch, while the subsiding branch is located over the cold patch. In the lower half of the CBL the subsiding branch diverges. As a result air parcels are horizontally advected towards the centre of the warm patch, where the flow converges and thermal energy accumulates. The bulk of the sensible heat is transported upwards within the uprising branch of the SC, which is evident by the positive values of the turbulent heat flux, while the turbulent heat flux is small or even negative in the remaining model domain. At the top of the CBL thermals that are associated with the uprising branch of the SC penetrate into the inversion layer. Due to the resulting prevailing compensatory subsidence directly beside the updraft region warm air is transported downwards, creating a region with negative turbulent heat flux. At the top of the CBL the uprising branch of the SC diverges and sensible heat is advected towards the cold patch. As a result of this warm air advection the upper CBL over the cold patch is stabilized (see also Fig. 1b), as discussed by Kang and Davis (2008). Over the centre of the cold patch the flow converges and the air subsides. The small or even negative values of the turbulent heat flux over the cold patch (e.g. for simulation A50L $32 *$ where the turbulent heat flux is negative above $0.4 z_{\mathrm{i}}$ ) indicate that the thermal convection is weakened or even completely suppressed by the presence of the SC. In order to verify that the SC suppresses thermal convection over the cold patch and to ensure that the low heat flux is not only a result of the lower surface heat flux, we performed homogeneous runs with identical surface heating as over the cold patch. The corresponding homogeneous runs are denoted as HOMA20C and HOMA50C for the small amplitude case A20L32* and the larger amplitude cases A50L32*, A50L08*, A50L16*, respectively. Figure 4c shows the profiles of the phase-averaged resolved-scale turbulent heat flux over the centre of the cold patch, as well as the domain-averaged resolved-scale heat flux for the homogeneous reference cases. It should be noted that for the heterogeneous cases the flux contribution by the SC is missing, however, it is obvious that the subsiding branch of the SC would not contribute to the vertical upward transport over the cold patch. For the HOMA20C and HOMA50C simulations the heat flux decreases linearly with height and exhibits a well-defined region with negative values at the top of the CBL, which is about 970 and $880 \mathrm{~m}$, respectively, whereas for the A20L32* and A50L32*, A50L08*, A50L16* simulations the turbulent heat flux over the cold patch decreases with height and is close to zero above 700 and $400 \mathrm{~m}$ (which is significantly lower than $z_{\mathrm{i}}$, see also Fig. 1), respectively. In addition, in order to be sure that turbulence is suppressed in the upper part of the CBL for the larger amplitude cases, we performed a further test simulation based on simulation A50L32*, where Lagrangian particles were released near the surface in the entire horizontal model domain and tracked for 30 min. Particle trajectories were calculated online by an embedded Lagrangian particle model according to Steinfeld et al. (2008). While a large number of particles reached the top of the CBL over the warm patch, no particle reached levels higher than $0.6 z_{\mathrm{i}}$ over the cold patch (not shown), confirming that the upper part of the CBL over the cold patch is decoupled from the surface heating below.

Figure 2 shows that the varying patch size and heat-flux amplitude affect the turbulent heat-flux pattern. For example, for simulation A50L32* the bulk of the turbulent upward 


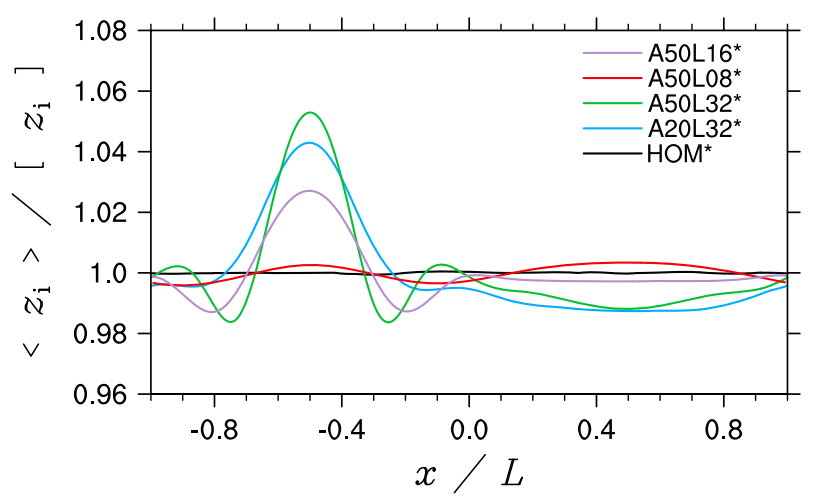

Fig. 3 Phase-averaged local boundary-layer depth $\left\langle z_{\mathrm{i}}(x)\right\rangle$ along the heterogeneous $x$-axis after $4 \mathrm{~h}$ of simulated time, normalized by the corresponding domain-averaged value. The $x$-axis is normalized by the respective patch size

transport is located in a thin tube-like pattern over the centre of the warm patch. For smaller patch size or heat-flux amplitude, e.g. for simulation A50L08* and A20L32*, the upward transport still occurs over the warm patch, but the tube-like pattern becomes wider relative to the patch size. Hence, the area where thermals penetrate into the inversion increases, and the area where the vertical upward transport is weakened by the SC decreases, which is particularly evident for simulation A50L08* by the region with positive and negative heat flux within the CBL.

Figure 3 shows the phase-averaged local boundary-layer depth $\left\langle z_{\mathrm{i}}(x)\right\rangle$ along the $x$-axis. As expected, no variation along the $x$-axis can be observed for the $\mathrm{HOM}^{*}$ simulation. The largest spatial variation can be observed for the A50L32* simulation, followed by the A20L32* and A50L16* simulations, while the spatial variation is much less pronounced for the A50L08* simulation. It is striking that the spatial variation for the A20L32* simulation is larger than for the A50L16* simulation, indicating that the spatial variation is more affected by the patch size than by the strength of the SC. The largest values of $\left\langle z_{\mathrm{i}}(x)\right\rangle$ occur over the centre of the warm patch. The A50L32* and A20L32* simulations exhibit a minimum over the cold patch, whereas simulation A50L08* exhibits a small maximum at this location. Even though the horizontal variations in $\left\langle z_{\mathrm{i}}(x)\right\rangle$ suggest horizontal variations in entrainment, it should be noted that $\left\langle z_{\mathrm{i}}(x)\right\rangle$ is not only determined by entrainment, but also the local structure of the interfacial layer (O11), as well as by encroachment (Maronga and Raasch 2013). Furthermore, the minima over the warm patch for simulation A50L32* and A50L16* are striking, which can be attributed to the penetration of thermals into the inversion and the resulting prevailing compensatory subsidence beside.

Figure 4a shows the domain-averaged total vertical sensible heat flux. As typical for the $\mathrm{CBL}$, the profiles decrease linearly with height. At the top of the CBL the profiles differ among the cases, which is shown in the close-up view in Fig. 4b. The area with negative heat flux provides a measure of entrainment (van Zanten et al. (1999)), which implies that the magnitude of entrainment correlates with the vertical depth of the region with negative flux and the magnitude of the minimum value (HA08). According to this assumption, the heat-flux profile for the A20L $32 *$ simulation suggests less entrainment compared to the HOM* simulation, whereas the profiles for the A50L32*, A50L08*, and A50L16* simulations suggest more entrainment. HA08 found similar sensible heat-flux profiles as shown in Fig. 4a, b. However, they argued that the heat-flux differences within the entrainment layer are rather an effect of 

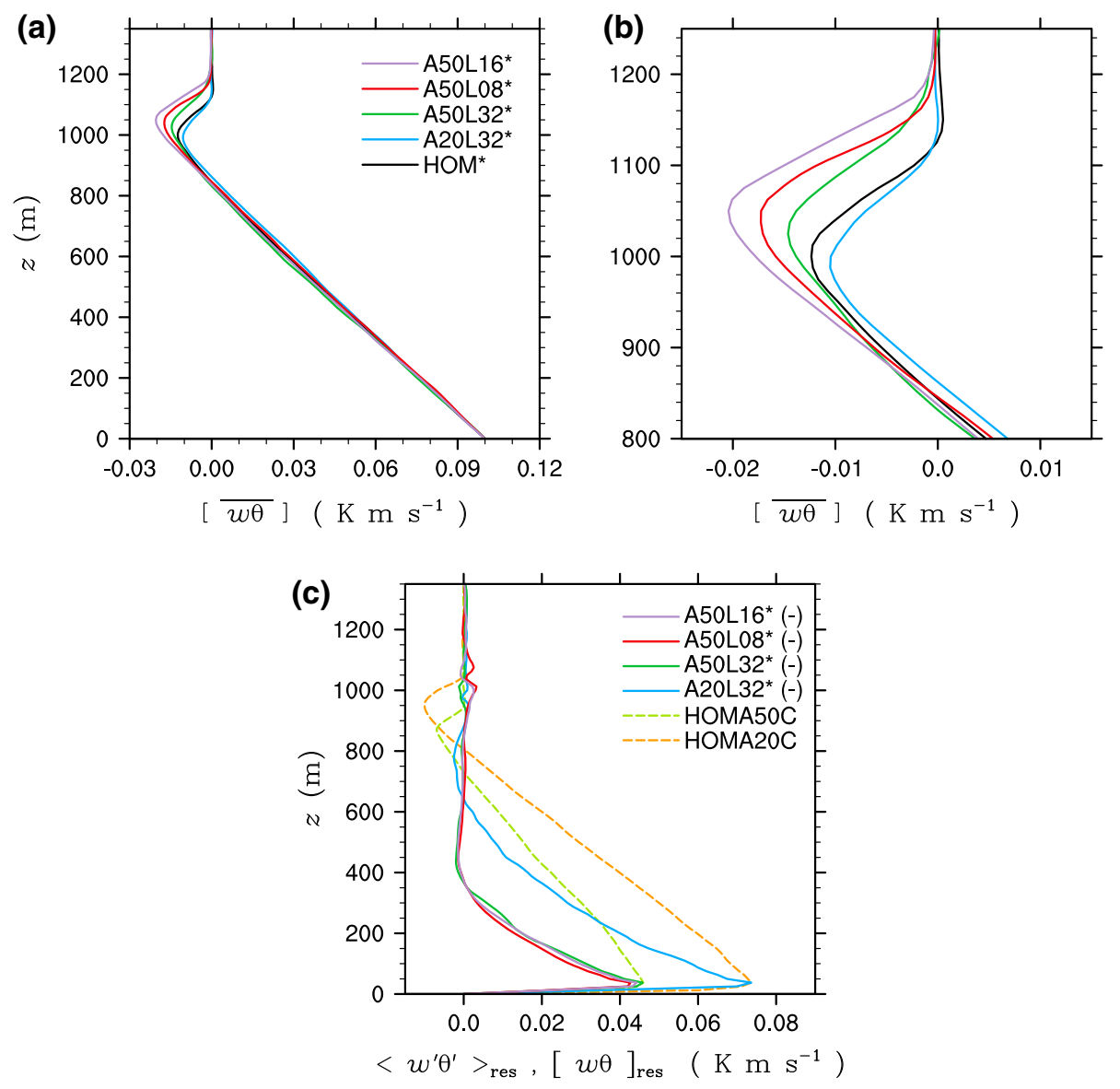

Fig. 4 a, b Vertical profiles of the domain-averaged total sensible heat flux after $4 \mathrm{~h}$ of simulated time, averaged over $15 \mathrm{~min}$, for $\mathbf{a}$ the entire CBL, and $\mathbf{b}$ for the entrainment zone. $\mathbf{c}$ Vertical profiles of the instantaneous phase-averaged (solid) resolved-scale sensible turbulent heat flux over the cold patch (minus), as well as of the domain-averaged (dashed) resolved-scale sensible heat flux for the homogeneous reference simulations HOMA20C and HOMA50C, after $4 \mathrm{~h}$ of simulated time. The surface heating in the HOMA20C and HOMA50C simulations is identical to the cold-patch surface heating in the small amplitude case A20L32* and the large amplitude cases A50L32*, A50L16*, A50L08*, respectively

horizontal averaging than the result of a heterogeneity-induced effect on entrainment. This is due to the fact that the vertical level and depth of the entrainment layer and entrainment itself vary horizontally, modifying the horizontally-averaged heat-flux profile, as already discussed in Sect. 1. In order to provide an entrainment measure that is independent from horizontal averaging, but gives local information that is required to improve understanding of how heterogeneous forcing affects entrainment, we introduce a new approach below.

\subsection{A New Approach to Determine Local Entrainment Rates}

Entrainment can be considered as a redistribution of heat where warm tropospheric air is mixed into the CBL through a stably-stratified layer, heating the CBL. This redistribution implies that a layer exists that is cooled by the same amount as the CBL is heated. In order to 


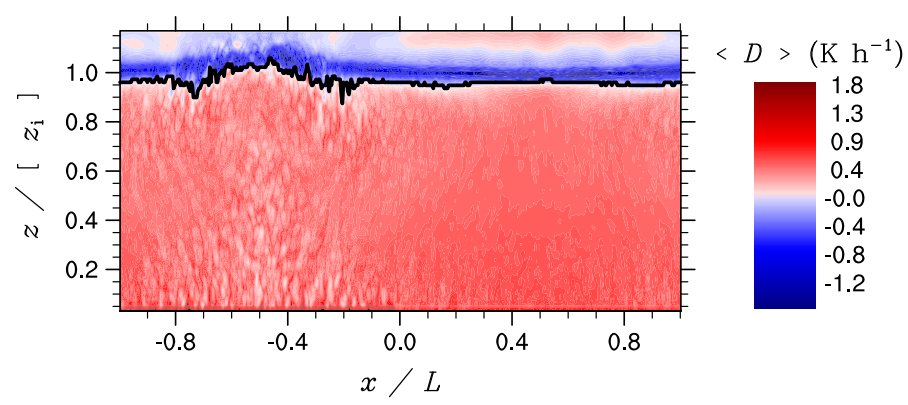

Fig. 5 Instantaneous vertical cross-section of $\langle D\rangle$ after $4 \mathrm{~h}$ of simulated time for simulation A50L32*. The black solid line indicates the zero-crossing according to our applied criteria. The $x$-axis is normalized by the patch size; the $z$-axis is normalized by the domain-averaged CBL depth

quantify the amount of heat entrained into the CBL, we consider the local temporal change of $\theta$ in our simulations, which is determined by

$$
\frac{\partial \theta}{\partial t}=-\frac{\partial u_{i} \theta}{\partial x_{i}}-\frac{\partial \tau_{i, \theta}}{\partial x_{i}}=D
$$

where the first term on the right-hand side describes the resolved-scale advection and the second term on the right-hand side is the parametrized turbulent transport on the subgrid scale. The prescribed surface heating given by $H_{0}(x)$ is considered in the subgrid-scale parametrization. To be consistent with the numerical discretization, we directly used the flux divergence provided by the advection scheme and the subgrid-scale parametrization. The sum of the right-hand side terms, which provides a heating or cooling rate at each grid point, is hereafter referred to as the total flux divergence $D$.

In order to investigate heterogeneity-induced differences in entrainment along the $x$ axis we applied phase-averaging on $D$. However, compared to the domain average, the phase average is statistically less significant because of the smaller number of statistically independent samples. To achieve statistically significant signals nevertheless, we extended the model domain along the $y$-axis for the selected cases as mentioned in Sect. 2.1 and shown in Table 1 . Moreover, to increase the number of statistically independent samples, we performed eight ensemble runs for each selected case, where each ensemble run had an individual development of the turbulent eddies but identical mean conditions, realized by imposing different initial random perturbations on the horizontal velocity fields.

Figure 5 shows a vertical cross-section of the phase- and ensemble-averaged instantaneous $\langle D\rangle$ after $4 \mathrm{~h}$ of simulated time for simulation A50L32*; $\langle D\rangle$ is positive within the CBL, indicating that the CBL becomes warmer due to surface heating and entrainment. Based on this heating rate only it is not possible to distinguish between warming of the CBL due to surface heating and due to the entrainment of warm air from the free atmosphere. Hence, we considered the cooling at the top of the CBL, indicated by the negative $\langle D\rangle$. This cooling is completely attributed to the redistribution of heat, where the cooler air from the CBL replaces the warmer air of the inversion, which is mixed into the CBL. Thus, this cooling of the lower part of the stably-stratified layer is equivalent to the heating of the CBL by entrainment, providing a direct and local measure of the entrained heat.

Entrainment events along the $y$-axis do not always take place at the same heights, hence, negative $\langle D\rangle$ appears within a region of a certain depth in the phase average. To incorporate all entrainment events and to obtain mean entrainment rates along the $y$-axis, we vertically integrated over the region with negative $\langle D\rangle$ 


$$
\langle E(x, t)\rangle=\int_{z_{1}(x, t)}^{z_{\mathrm{u}}(x, t)} \mathrm{d} z\langle D(x, z, t)\rangle,
$$

where $z_{1}(x, t)$ and $z_{\mathrm{u}}(x, t)$ are the lower and upper integration bounds. On closer inspection Fig. 5 reveals isolated negative values of $\langle D\rangle$ below the continuous region with negative values, e.g. at $x=-0.8 \mathrm{~L}$, which might also belong to entrainment events. The vertical integration including these isolated negative values leads to almost identical results when we integrated over the continuous region with negative values neglecting these isolated values. Hence, starting from the surface, we defined $z_{1}(x, t)$ (indicated by the black solid line in Fig. 5) as the height where $\langle D\rangle$ reaches negative values and does not change its sign the four grid points above. Figure 5 shows non-zero values of $\langle D\rangle$ within the free atmosphere with alternating sign, which can be attributed to gravity waves and weak compensating motions that do not necessarily belong to entrainment processes. For this reason it is sometimes difficult to decide whether a cooling within the free atmosphere belongs to an entrainment event or not. However, a sensitivity analysis (not shown) revealed $\langle E\rangle$ almost independent of the choice of the upper integration bound, so that we decided to integrate up to the top of the model domain.

We calculated $\langle E\rangle$ every $60 \mathrm{~s}$ during the third and fourth hour of the simulation. Subsequently, we applied a temporal average to these values. Domain-averaged entrainment rates were obtained from the time-averaged values by averaging them along the $x$-direction.

It is obvious that the calculation of $\langle E\rangle$ requires a well-defined region with negative $\langle D\rangle$. Hence, the sample size of the combined phase and ensemble average should be large enough to allow for identifying a continuous region with negative $\langle D\rangle$ at each position along the $x$-direction. More precisely, we used the internal error expression of Lenschow et al. (1994) based on the integral length scale of $D$ to estimate the required sample size (i.e. the averaging length along the $y$-direction). As the sampling error depends on the inverse of the averaging length, we have chosen the averaging length such that the relative error of the combined ensemble and phase average was $<5 \%$ in the entrainment and mixed layers. This again illustrates the requirement of ensemble averaging and an extended model domain in the $y$-direction (see Table 1).

This new method allows for separating the CBL warming (and thus growth) into contributions from the entrainment of warm air and from surface heating, i.e. encroachment, which is discussed in Sect. 3.3. The encroachment rate was calculated by integrating $\langle D\rangle$ from the surface to the top of the model domain (which is equivalent to subtracting $\langle E\rangle$ from the integral of $\langle D\rangle$ from the surface to $\left.z_{1}(x, t)\right)$.

\subsection{Domain-Averaged Entrainment Rates and Comparison with Other Methods}

In order to evaluate our introduced approach, we compared the temporally and domainaveraged entrainment rates $\overline{[E]}$ with commonly used entrainment measures. Therefore, we calculated $w_{\mathrm{e}}$ between the third and fourth hour of the simulation. Moreover, we calculated the vertical integral of domain- and time-averaged total sensible heat-flux profiles

$$
I_{w \theta}=\frac{g}{\theta_{0}} \int \mathrm{d} z \overline{[w \theta]}
$$

over the region where $\overline{[w \theta]}<0 ; g$ denotes the acceleration due to gravity, $\theta_{0}$ is the basic state in the Boussinesq equations; $g / \theta_{0}$ was identical in all simulations. $I_{w \theta}$ describes the net 
Table 2 Physical unit and interpretation of different entrainment measures

\begin{tabular}{lll}
\hline Entrainment measure & Unit & Physical interpretation \\
\hline$w_{\mathrm{e}}$ & $\mathrm{m} \mathrm{s}^{-1}$ & CBL growth by encroachment and entrainment \\
$I_{w \theta}$ & $\mathrm{m}^{3} \mathrm{~s}^{-3}$ & $\begin{array}{l}\text { Rate of net TKE consumption in the entrainment layer, } \\
\text { associated with the change of thermal energy in the mixed } \\
\end{array}$ \\
& $\begin{array}{l}\text { layer by entrainment; } \\
\text { might be affected by horizontal averaging }\end{array}$ \\
& $\mathrm{K} \mathrm{m} \mathrm{s}^{-1}$ & $\begin{array}{c}\text { Amount of thermal energy transported from the free } \\
\text { atmosphere into the CBL per unit area }\end{array}$ \\
\end{tabular}

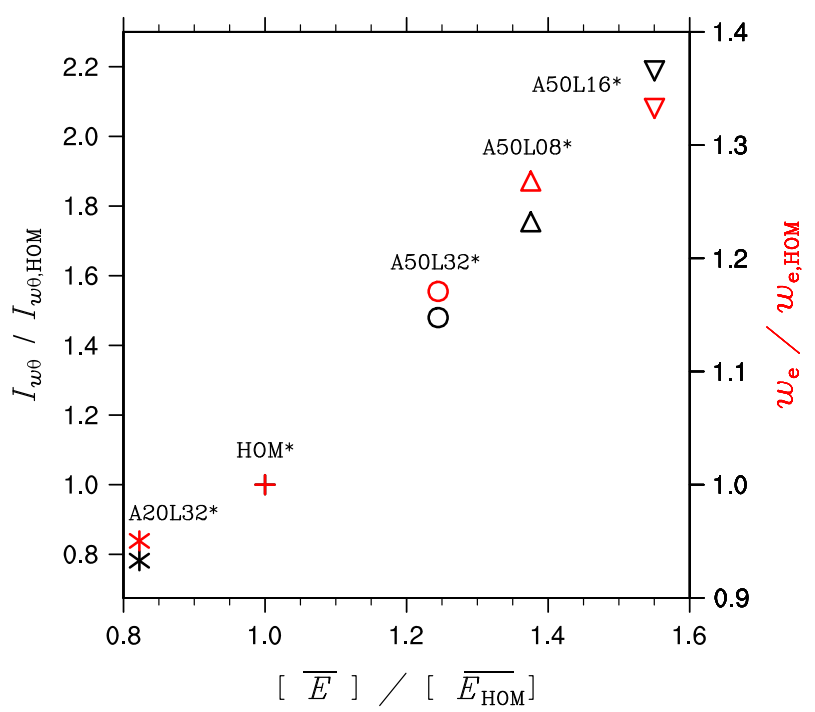

Fig. 6 Comparison of $\overline{[E]}$ with $I_{w \theta}$ (black symbols) and $w_{\mathrm{e}}$ (red symbols). Please note the different scale between the left and the right ordinate axes. All values are normalized with the corresponding value of the $\mathrm{HOM}^{*}$ simulation

rate of TKE consumption by entrainment (Stull 1976). The different physical interpretations and units of entrainment measures used in this study are listed in Table 2.

In order to compare dimensionless quantities we normalized the respective values by the corresponding value of the $\mathrm{HOM}^{*}$ simulation. Figure 6 shows that $\overline{[E]}$ correlates with $I_{w \theta}$ and $w_{\mathrm{e}}$ for the simulated cases. It is striking that $\overline{[E]}, I_{w \theta}$ and $w_{\mathrm{e}}$ vary significantly among the selected cases, where $\overline{[E]}$ is about $20 \%$ smaller for the A20L $32 *$ simulation compared to the HOM* simulation and about 30, 40, and even $60 \%$ larger for the A50L32*, A50L08*, and A50L16* simulations, respectively. The underlying physics of this heterogeneity-induced impact are discussed in the following sections. $\overline{[E]}$ correlates almost linearly with $I_{w \theta}$, revealing that the variations in the heat flux within the entrainment layer cannot be attributed only to the effect of horizontal averaging as stated by HA08. Nevertheless, due to the fact that the relative differences among the cases are larger in $I_{w \theta}$ than in $\overline{[E]}$, we do not rule out that horizontal averaging can have an effect on $I_{w \theta}$, where cases with larger heat-flux amplitude have a deeper region with negative flux. 


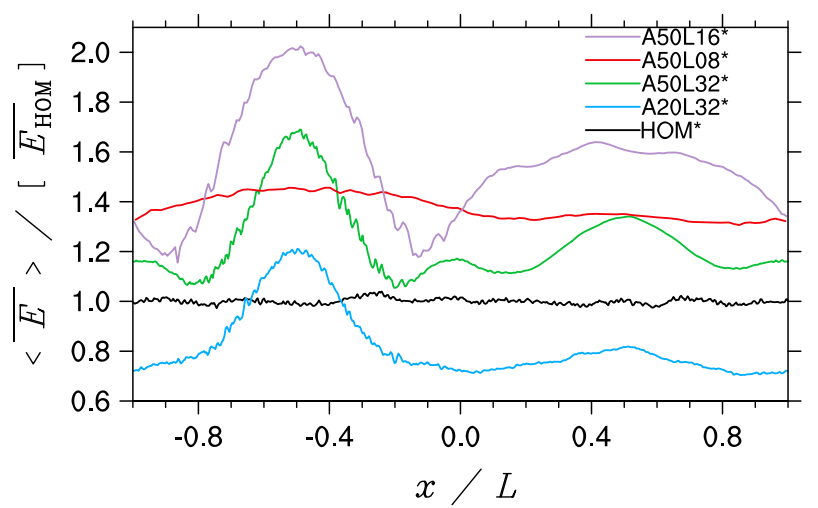

Fig. 7 Horizontal distribution of $\langle\overline{E(x)}\rangle$ along the heterogeneous $x$-axis, temporally averaged over $1 \mathrm{~h}$ and normalized by the domain-averaged value of the $\mathrm{HOM}^{*}$ simulation. The $x$-axis is normalized by the patch size

$\overline{[E]}$ correlates linearly with $w_{\mathrm{e}}$, i.e. the CBL grows faster for cases with larger $\overline{[E]}$ and vice versa. Even though both methods give the same qualitative result, it is striking that the relative differences among the cases are smaller in $w_{\mathrm{e}}$ than in $\overline{[E]}$. This is because $w_{\mathrm{e}}$ is dominantly determined by encroachment and thus less sensitive to variations in entrainment.

We quantified the contribution of entrainment and encroachment on the boundary-layer evolution. As expected, encroachment was identical in all simulations. The relative contribution of entrainment to the total CBL heating was 10, 12, 15, 20 and $23 \%$ for the A20L32*, $\mathrm{HOM}^{*}, \mathrm{~A} 50 \mathrm{~L} 32 *, \mathrm{~A} 50 \mathrm{~L} 08^{*}$ and A50L16* simulations, respectively, while the remaining relative contribution of encroachment was $90,88,85,80$ and $77 \%$ for the A20L32*, HOM*, A50L32*, A50L08* and A50L16* simulations, respectively. The obtained relative contributions are in the range of the values given in the literature (e.g. Stull 1976; Canut et al. 2010), further indicating that the new method gives reliable estimates of entrainment and encroachment.

\subsection{Local Entrainment Rates}

The new local entrainment measure now allows us to study the effect of heterogeneity on the local entrainment rate. Figure 7 shows $\langle\overline{E(x)}\rangle$, normalized by the domain-averaged value of the $\mathrm{HOM}^{*}$ simulation. As expected, $\langle\overline{E(x)}\rangle$ is homogeneously distributed for the HOM* simulation, whereas it varies along the $x$-axis for the heterogeneous simulations. The largest entrainment occurs over the centre of the warm patch, where thermal energy is accumulated and strong thermals can penetrate rigorously into the inversion layer. For simulation A50L32* and A50L16* two minima over the warm patch can be observed; these minima can be attributed to the prevailing compensatory subsidence beside the thermal updrafts, as already shown for the spatial distribution of $\left\langle z_{\mathrm{i}}(x)\right\rangle$ (see Sect. 3.1). As discussed in Sect. 3.1, the width of the uprising branch of the SC increases relative to the patch size for decreasing patch sizes, so that the effective area over which the thermal convection can penetrate into the capping inversion enlarges and more uniformly distributed values of $\langle\overline{E(x)}\rangle$ can be observed over the warm patch, which is particularly evident for simulation A50L08*. Except for simulation A50L08*, a second maximum can be observed over the cold patch. This is surprising since almost no thermals reach the inversion layer over the cold patch, triggering entrainment events. We discuss the possible mechanisms that might explain this finding in Sect. 3.5. Moreover, it is surprising that the A20L32* simulation shows less entrainment than 


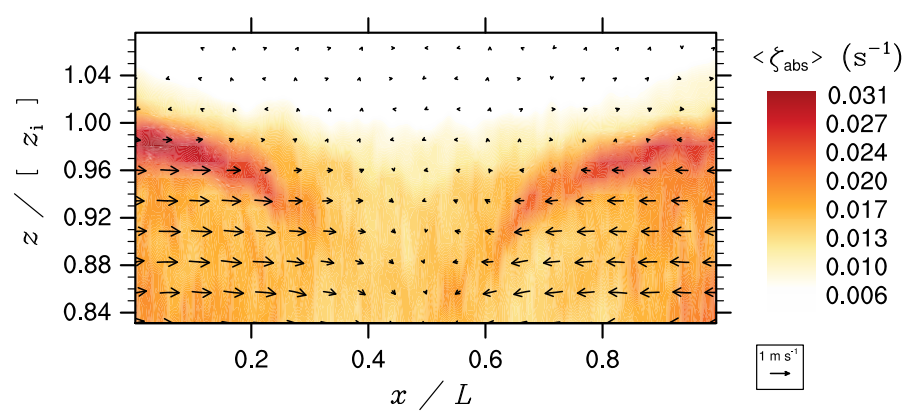

Fig. 8 Instantaneous vertical cross-section of $\left\langle\zeta_{\text {abs }}\right\rangle$ at the top of the CBL over the cold patch for simulation A50L32* after $4 \mathrm{~h}$ of simulated time. The phase-averaged flow is displayed by the wind vectors. The $x$-axis is normalized by the patch size; the $z$-axis is normalized by the domain-averaged CBL depth

the $\mathrm{HOM}^{*}$ simulation, in contrast to the larger heterogeneity amplitude cases, even though $\langle\overline{E(x)}\rangle$ is similarly distributed. The possible underlying physics of this effect are discussed in Sect. 3.6.

Based on the local distribution of $\left\langle z_{\mathrm{i}}(x)\right\rangle$, O11 inferred that entrainment over the cold patch is reduced. Even though $\left\langle z_{\mathrm{i}}(x)\right\rangle$ and $\langle\overline{E(x)}\rangle$ (compare Figs. 3 and 7) correlate fairly well over the warm patch, including the minimum values next to the updraft region, they do not correlate over the cold patch, where $\langle\overline{E(x)}\rangle$ has a maximum but $\left\langle z_{\mathrm{i}}(x)\right\rangle$ exhibits a minimum. This shows that the spatial distribution of $\left\langle z_{\mathrm{i}}(x)\right\rangle$ is not a reliable measure of local entrainment. Hence we disagree with $\mathrm{O} 11$ and argue that the spatial distribution of $\left\langle z_{\mathrm{i}}(x)\right\rangle$ is determined by encroachment and by the local structure of the interfacial layer rather than by entrainment.

\subsection{Entrainment Physics over the Cold Patch}

In order to investigate the underlying physics of the unexpected large entrainment rate over the cold patch, we analyzed the local turbulence intensity, provided by means of $\zeta_{\text {abs }}$ (see Sect. 2.2). The instantaneous phase-averaged $\left\langle\zeta_{\text {abs }}\right\rangle$ over the cold patch is shown in Fig. 8 for the A50L32* simulation after $4 \mathrm{~h}$ of simulated time. Within the free atmosphere $\left\langle\zeta_{\mathrm{abs}}\right\rangle$ is close to zero, while larger values can be observed within the CBL, and in particular at its top, where entrainment takes place. It is striking that the heterogeneity-induced mean flow exhibits a vertical shear at the interfacial layer, indicated by the wind vectors. This local shear occurs mainly at the edges of the cold patch, where it contributes to the larger values of $\left\langle\zeta_{\text {abs }}\right\rangle$ that can be observed at these locations. Moreover, flow visualization revealed horizontally advected turbulence to be an important factor increasing $\left\langle\zeta_{\text {abs }}\right\rangle$ at the edges of the cold patch (not shown). Due to the absence of thermal convection as shown in Fig. 2, we hence suppose that entrainment at the edges of the cold patch is mainly driven by the vertical wind shear and the horizontally advected turbulence. Towards the centre of the cold patch $\left\langle\zeta_{\text {abs }}\right\rangle$ decreases gradually, indicating only weak turbulence there. This is due to the fact that the horizontally advected turbulence dissipates gradually towards the centre of the cold patch and the local vertical wind shear vanishes due to the horizontal flow convergence. However, the weak turbulence over the centre of the cold patch conflicts with the large entrainment that we observed there (see Fig. 7).

In order to examine how the converging SC over the cold patch interacts with the inversion layer, we visualized the flow to identify possible mechanisms causing the large entrainment. Figure 9 shows an example of the temporal evolution of entrainment events over the centre 
(a) t' $=10925 \mathrm{~s}$

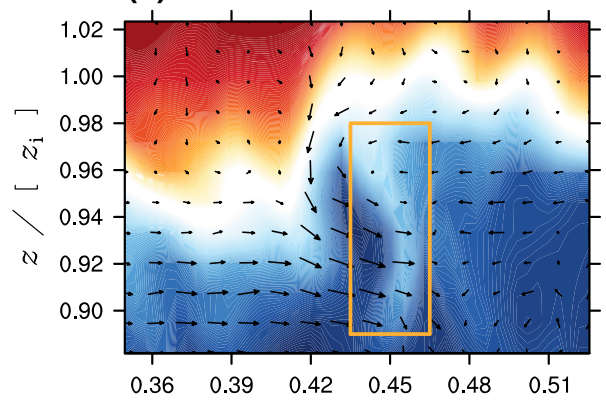

(c) $\mathrm{t}=\mathrm{t}^{\prime}+93 \mathrm{~s}$

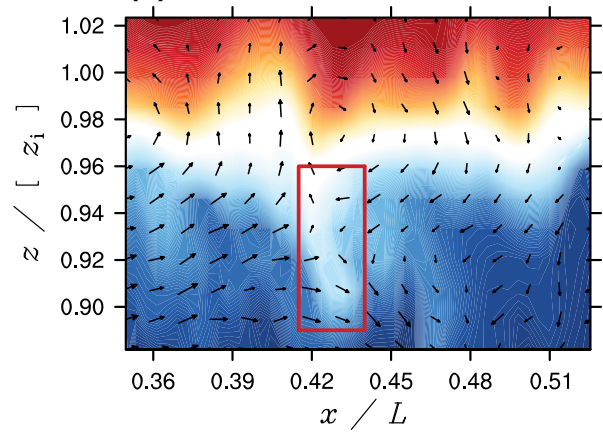

$\theta(\mathrm{K})$ (b) $t=t^{\prime}+63 \mathrm{~s}$

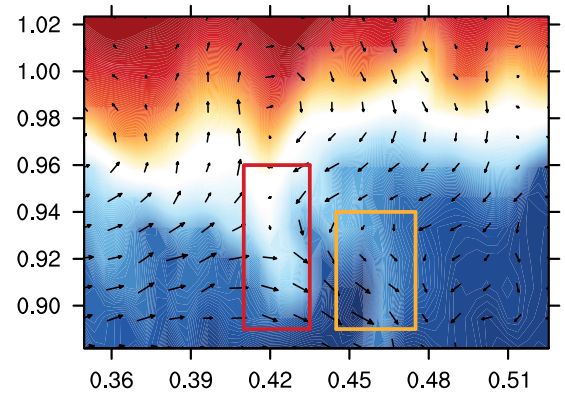

(d) $\mathrm{t}=\mathrm{t}^{\prime}+160 \mathrm{~s}$

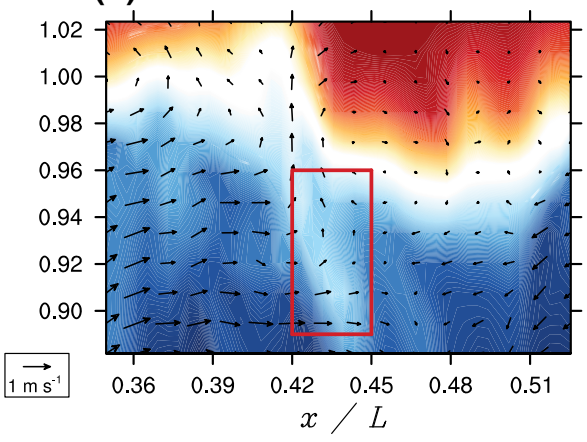

294.6294 .8295 .0295 .1295 .3295 .5295 .7295 .9296 .0

Fig. 9 Instantaneous vertical cross-sections of $\theta$ at the top of the CBL illustrating the temporal evolution of entrainment events over the centre of the cold patch for simulation A50L32*. Additionally, the corresponding instantaneous flow is displayed by the wind vectors. The $x$-axis is normalized by the patch size; the $z$-axis is normalized by the domain-averaged CBL depth. The orange and red boxes highlight and track pockets of warm air that dip down into the CBL

of the cold patch for the A50L32* simulation by four instantaneous snapshots of $\theta$ and the corresponding flow field. It is striking that the interfacial layer is wrinkled, as it can be observed e.g. by the pocket of warm air that dips down into the CBL at $x=0.45 \mathrm{~L}$ in Fig. 9a, indicated by the orange box. In Fig. $9 \mathrm{~b}$ this pocket of warm air is pinched off and separated from the inversion layer, while it becomes part of the mixed layer in the following due to turbulent mixing. Meanwhile, another wrinkle has been developed at $x=0.42 \mathrm{~L}$ in Fig. 9b, indicated by the red box, which results from the strong downdraft that can be observed at $x=0.42 \mathrm{~L}$ in Fig. 9a. This pocket of warm air deepens and narrows in the following, while the cooler air from the mixed layer starts to pinch off the pocket of warmer air at its upper part (see Fig. 9c). Subsequently, this warmer air is further drawn into the CBL and mixed with the ambient cooler air (see Fig. 9d). Sullivan et al. (1998) showed a similar entrainment mechanism where pockets of warm air dipped down into the CBL and were pinched off by strong compensating horizontal motions. However, in contrast to the entrainment events observed by e.g. Sullivan et al. (1998) or Träumner et al. (2011) that were triggered by thermal convection, the physical reason for the observed entrainment events over the cold patch is a different one in the present study, since the thermal convection is completely suppressed.

It is obvious that the local height of the interfacial layer varies horizontally, as can be observed by the step-like structures of the interfacial layer where strong horizontal temper- 
ature gradients occur, particularly in Fig. 9a, d. These step-like structures are related to the horizontal advection of eddies towards the centre of the cold patch (also horizontal propagating gravity waves might be important), where they collide and interact with each other, which results in local distortions of the interfacial layer. It is striking that at the edge of these step-like structures strong vertical compensating motions occur, e.g. at $x=0.42 \mathrm{~L}$ in Fig. 9a, d. As a result of these compensating motions, warm air is drawn in the CBL forming wrinkles if the motion is directed downward as e.g. in Fig. 9a, or the step-like feature is further developed if the motion is directed upward as e.g. in Fig. 9d. Owing to the fact that we observed (by visual flow inspection only) such distinct step-like structures and wrinkles primarily over the centre of the cold patch, we hypothesize that the wrinkling of the interfacial layer and the resulting entrainment events are mainly caused by the horizontal flow convergence.

Even though the turbulence intensity over the centre of the cold patch is smaller, entrainment is larger compared to e.g. the edges of the cold patch, where increased turbulence but less entrainment can be observed. We suppose that this might be related to the prevailing subsidence over the centre of the cold patch that counteracts the positive buoyancy of the drawn-in pockets of warm air, and therefore increases the possibility of entrainment of this warm air.

These processes that trigger entrainment over the cold patch are assumed to depend on the strength of the SC. For instance, a stronger SC is accompanied by more shear and advection, as well as a stronger flow convergence. Moreover, the horizontal scale of the SC might also affect the flow convergence. For smaller length scales, i.e. smaller patch sizes, the flow decelerates over shorter distances compared to larger length scales. As a result, the flow convergence becomes stronger, which could explain the larger values of $\langle\overline{E(x)}\rangle$ that can be observed for the A50L16* and A50L08* simulations over the cold patch, compared to the A50L32* simulation.

Although we discussed and demonstrated the possible underlying processes of the heterogeneity-induced effect on entrainment over the cold patch, we could not quantify the relative importance of each of them and how they scale with the strength of the SC. This would require a detailed investigation of local balance equations for the TKE and the heat flux within the entrainment layer, which would be challenging since it would require that both the mesoscale and the turbulent contributions be included.

\subsection{What Causes the Reduced Entrainment for Small Heterogeneity Amplitudes?}

From Fig. 6 we note that the A20L32* simulation on average exhibits less entrainment than the $\mathrm{HOM}^{*}$ simulation, even over the warm patch (see Fig. 7). This is in contrast to the other heterogeneous cases and indicates the presence of a process counteracting the thermal convection over the warm patch. In the lower CBL the horizontal branches of the SC are directed towards the centre of the warm patch. On the one hand, due to the horizontal flow convergence thermal energy that originate mainly from the warm-patch surface heating is aggregated over the centre of the warm patch, strengthening the thermal convection at this location. On the other hand, the SC advects air parcels from the cold towards the warm patch which are on average cooler than the surrounding air (see Fig. 1), stabilizing the lower CBL over the warm patch, as shown by Kang and Davis (2008) and Kang et al. (2012). For the A20L32* simulation we suppose that the stabilization is the governing mechanism reducing entrainment over the warm patch, whereas e.g. for the A50L32* simulation the aggregation of thermal energy dominates, increasing entrainment over the warm patch. We note that the balance of these competing physical processes, i.e. whether the stabilization is able to 
weaken thermal convection or not, and their dependence on the strength of the SC, is not well understood so far and need to be further explored.

Figure 1 indicates that the upper CBL over the cold patch is also stabilized, which results from the horizontal warm air advection by the SC in the upper CBL towards the cold patch (Kang and Davis 2008). In particular for the A20L32* simulation, where weak thermal convection still reaches the top of the CBL over the cold patch, this stabilization further weakens the thermal convection, reducing entrainment over the cold patch. For the larger heatflux amplitude cases we suppose that the stabilization of the upper CBL over the cold patch is of minor importance for entrainment as thermal convection is already fully suppressed.

\subsection{Temporal Evolution of the CBL Depth—Dependence on Heat-Flux Amplitude, Patch Size and Mean Wind Speed}

In order to provide a more comprehensive picture of how surface heterogeneities affect entrainment, we investigated a wider range of heterogeneity amplitudes and patch sizes, as well as wind speeds and wind directions. Due to the large computational effort to determine the entrainment rate by means of the local flux divergence, we used the temporal evolution of $\left[z_{\mathrm{i}}\right]$ instead, which allowed us to use smaller-domain simulations (see Table 1). As the temporal evolution of $\left[z_{\mathrm{i}}\right]$ is primarily determined by encroachment, we normalized the respective values of $\left[z_{\mathrm{i}}\right]$ by the values of the HOM simulation at the corresponding point in time, in order to remove the identical contributions from encroachment to emphasize the differences in entrainment. Figure 10a shows the normalized temporal evolution of $\left[z_{\mathrm{i}}\right]$ for different heterogeneity amplitudes. During the first hour, where the CBL was heated homogeneously in all simulations, the temporal evolution of $\left[z_{\mathrm{i}}\right]$ is identical. After $1 \mathrm{~h}$ of simulated time, the heterogeneous surface heating starts and the curves begin to diverge. Particularly larger amplitude runs show a local maximum between the second and third hour of the simulation. This maximum is linked to periodic oscillations of the SC strength found by Letzel and Raasch (2003), which remain for the entire period of the simulation, where the horizontal pressure and temperature gradients have to reach a quasi-steady state. For the A10L32 and A20L32 simulations the CBL grows slightly slower compared to the HOM simulation, consistent with Patton et al. (2005) and O11 for similar heterogeneity amplitudes and patch sizes. For A30L32 and larger amplitudes the CBL grows more rapidly compared to the HOM simulation, while the fastest growth can be observed for the largest amplitudes, consistent with Avissar and Schmidt (1998), Raasch and Harbusch (2001) and O11. We note that the effect of heterogeneity on entrainment is in contrast to HA08, who found, by means of the temporal evolution of $\left[z_{\mathrm{i}}\right]$, no indication that entrainment is affected by heterogeneous surface heating. However, HA08 used a relative short analysis period, making it difficult to detect different temporal evolutions of $\left[z_{\mathrm{i}}\right]$, particularly for non-normalized time series, which might explain their different observation.

Figure $10 \mathrm{~b}$ shows how the temporal evolution of $\left[z_{\mathrm{i}}\right]$ depends on the patch size. The largest impact on domain-averaged entrainment can be observed for simulations A50L08 and A50L16 with patch sizes in the order of $\left[z_{\mathrm{i}}\right]$. For larger patch sizes the effect of the surface heterogeneity on entrainment decreases. Despite the strong oscillations that can be observed for simulations A50L64 and A50L96, the temporal mean growth of the CBL is close to the HOM simulation for both cases, which is in agreement with the findings reported by $\mathrm{O} 11$. They argued that for patch sizes significantly larger than $\left[z_{\mathrm{i}}\right]$ the impact of the SC decreases and is only of minor importance for entrainment. In this case the boundary layer over the warm and the cold patches become separated and local entrainment depends more on the surface heating of the respective patch than on the strength of the SC, as also recently shown by 
(a)

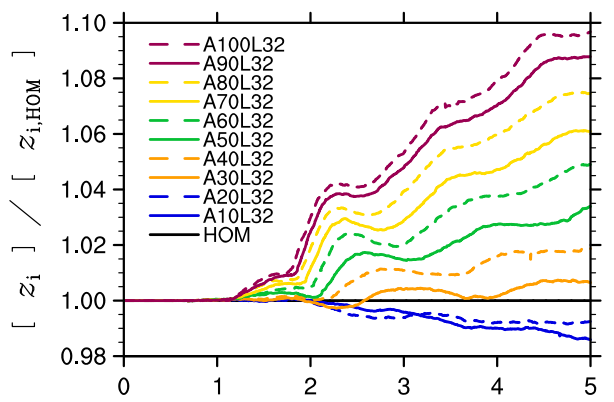

(c)

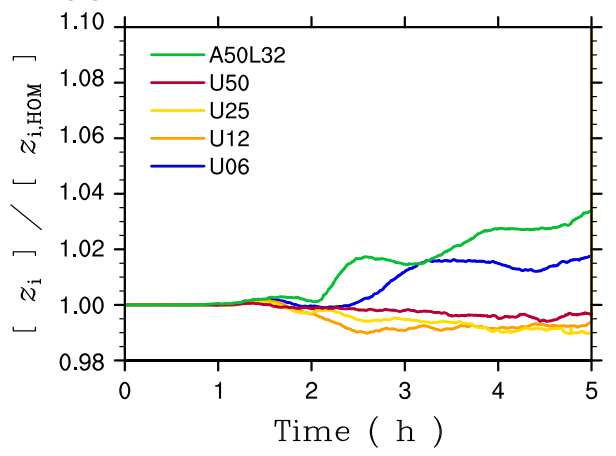

(b)

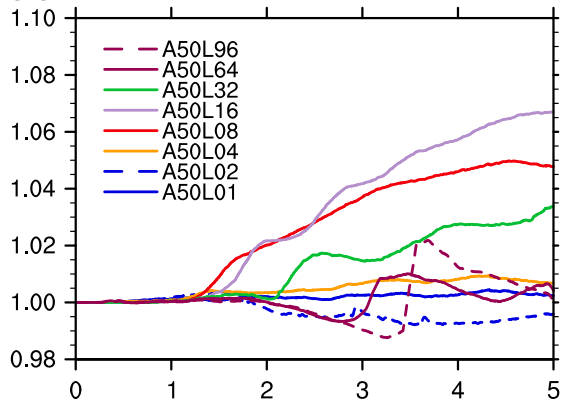

(d)

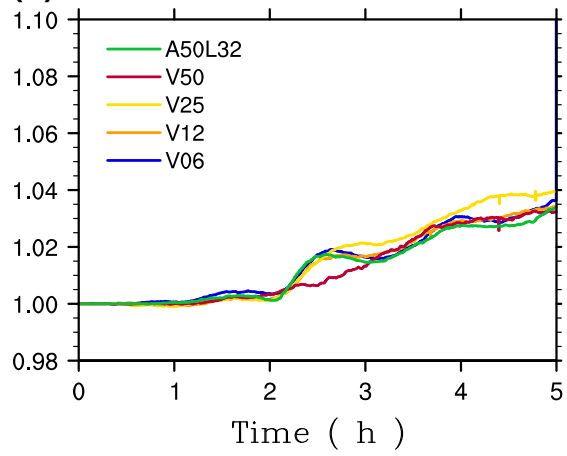

Fig. 10 Temporal evolution of $\left[z_{\mathrm{i}}\right]$, normalized by the respective value of the homogeneous reference simulation at the corresponding point in time, for a varying heterogeneity amplitude, b varying patch size, $\mathbf{c}$ varying wind speed with wind perpendicular to the border between the patches, and $\mathbf{d}$ varying wind speed with wind parallel to the border between the patches. For the sake of comparison the heterogeneous reference case A50L32 is displayed in all plots

Sühring and Raasch (2013) for the larger heterogeneity scales of the LITFASS-2003 terrain. We note that the decreased heterogeneity impact on entrainment for larger patch sizes is in contrast to the findings reported by Avissar and Schmidt (1998), who found that entrainment further increases for increasing patch size (up to $40 \mathrm{~km}$ ). However, Avissar and Schmidt (1998) inferred information on entrainment form vertical profiles of the sensible heat flux only. For a similar set-up Letzel and Raasch (2003) showed that heat-flux profiles are strongly influenced by the temporal oscillation of the SC, so that the results reported by Avissar and Schmidt (1998) concerning entrainment should be interpreted carefully. Furthermore, we note that the dependence of entrainment on patch size shown in Fig. 10b differs from the results shown by Patton et al. (2005), who found the largest impact on entrainment for significantly larger patch sizes (about $9 z_{\mathrm{i}}$ ). This might be due to the less pronounced heat-flux amplitude in their study, suggesting that the effect of varying patch size on entrainment might interact with the effect of varying heat-flux amplitude.

For patch sizes significantly smaller than $\left[z_{\mathrm{i}}\right]$ the effect on the CBL growth is small. For the A50L01 simulation the impact is negligible, while $\left[z_{\mathrm{i}}\right]$ is slightly smaller for A50L02 and slightly larger for the A50L04 simulation. Even though the surface-heterogeneity signal blends within the CBL and no SC can be observed (not shown), this suggests that also small-scale surface heterogeneities affect entrainment.

It is striking that the curve for the A50L08 simulation flattens and even drops down after about $4 \mathrm{~h}$ of simulated time. After this point in time the largest eddies within the CBL are large 
(a)

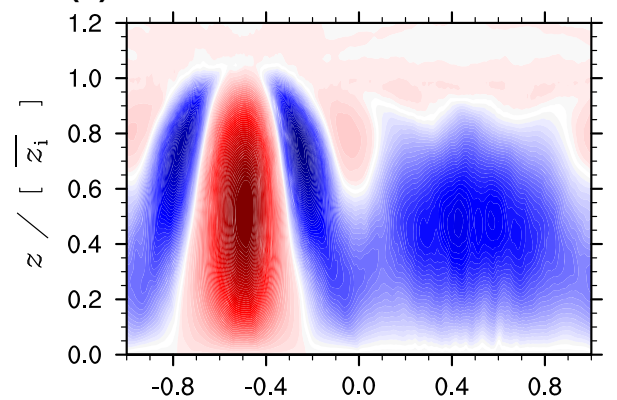

(c)

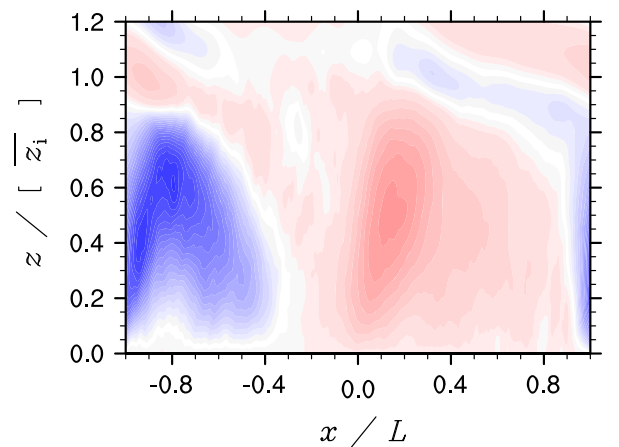

(b)

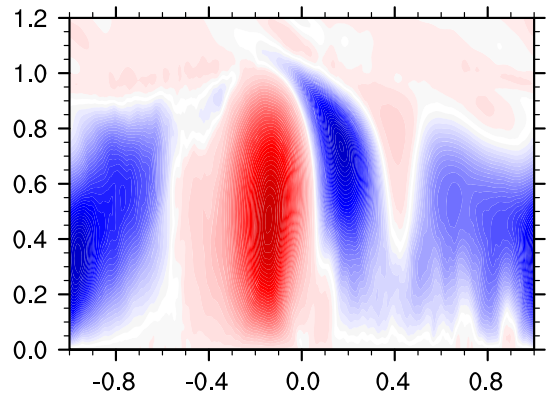

(d)

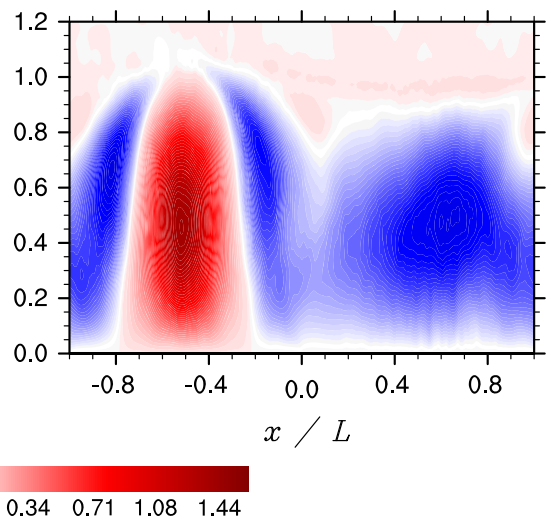

Fig. 11 15-min time-averaged vertical cross-section of the phase-averaged vertical velocity, indicating the strength of the SC, for the a A50L32, b U06, c U25, and d V25 simulations after $4 \mathrm{~h}$ of simulated time. The $x$-axis is normalized by the respective patch size; the $z$-axis is normalized by the respective domain- and time-averaged CBL depth

enough to efficiently reduce the horizontal temperature gradients that trigger the SC, so that the SC breaks down. Therefore, the SC-induced processes that are increasing entrainment vanish and entrainment decreases immediately.

Figure 10c, d shows the effect of surface heterogeneities on the temporal evolution of $\left[z_{i}\right]$ for varying wind speed and wind direction. We normalized the respective values of $\left[z_{\mathrm{i}}\right]$ by the values of the respective homogeneous run (with identical geostrophic forcing) at the corresponding point in time, in order to separate the effect of surface heterogeneity on entrainment from the effect of varying wind shear. For simulation U06, where the flow is aligned perpendicular to the border between the patches, the CBL grows faster than the respective homogeneous case. However, compared to the A50L32 simulation, which is the corresponding case without mean wind, the heterogeneity-induced impact on entrainment is reduced. Raasch and Harbusch (2001) found that signals from the surface heterogeneity smear out as the increased horizontal mixing by the mean wind reduces the temperature gradients and thus weakens the corresponding SC. This is shown in Fig. 11b, where the uprising branch of the SC for U06 is less pronounced compared to the A50L32 simulation, shown in Fig. 11a, and shifted in downwind direction towards the cold patch. Figure 10c shows even slightly smaller values of $\left[z_{i}\right]$ for the U12 and U25 simulations compared to the respective homogeneous case. Since the SC is only weakly pronounced for these cases (see Fig. 11c), we suppose that the weak SC counteracts the thermal convection due to the 
stabilization effect as discussed in Sect. 3.6. For further increasing wind speed the SC breaks down completely and no significant effect of heterogeneity on the boundary-layer evolution can be observed for the U50 simulation, confirming the results shown by $\mathrm{O} 11$.

If the flow is aligned parallel to the border between the patches the heterogeneity signal is not smeared out so that the horizontal temperature and pressure gradients persist, which results in similar SCs such as in the A50L32 simulation, as shown for example in Fig. 11d for simulation V25. Hence, the heterogeneity-induced effect on entrainment persists, leading to a similar CBL growth as in simulation A50L32.

\section{Summary}

In this study we investigated the impact of one-dimensional stripe-like surface heat-flux heterogeneities on mixed-layer top entrainment. The temporal evolution of $\left[z_{\mathrm{i}}\right]$ revealed a significant effect of surface heterogeneity on entrainment, depending on heat-flux amplitude, patch size, as well as wind speed and wind direction. For small heat-flux amplitudes with patch sizes in the order of $z_{i}$ we found slightly decreased entrainment compared to the homogeneous case, in agreement with Patton et al. (2005) and O11, while we found increased entrainment for large heat-flux amplitudes, in agreement with Avissar and Schmidt (1998), Raasch and Harbusch (2001) and O11. Thus, we could disprove that entrainment is not affected by the heterogeneous surface heating as stated by HA08. The largest increase in entrainment could be observed for patch sizes in the order of $z_{\mathrm{i}}$. For patch sizes significantly smaller than $z_{\mathrm{i}}$ the heterogeneity effect on entrainment is small, but we nevertheless observed slightly modified entrainment compared to the homogeneous case. Even though the heterogeneity signal is blended within the CBL in this case, our results suggest that also small-scale surface heterogeneities affect the mixed-layer top entrainment. For patch sizes significantly larger than $z_{\mathrm{i}}$ entrainment decreases with increasing patch size, approaching the homogeneous case, consistent with O11. We note that this finding is in contrast to Avissar and Schmidt (1998), who found that entrainment further increases for increasing patch size. However, Avissar and Schmidt (1998) inferred information on entrainment form vertical profiles of the sensible heat flux only. For a similar set-up Letzel and Raasch (2003) showed that heat-flux profiles are strongly influenced by the temporal oscillation of the SC, so that the results reported by Avissar and Schmidt (1998) concerning entrainment should be interpreted carefully. Furthermore, we note that the dependence of entrainment on patch size in this study is different compared to the results shown by Patton et al. (2005), who found the largest increase in entrainment for significantly larger patch sizes of about $9 z_{\mathrm{i}}$, whereas our results show the largest increase for patch sizes of about $z_{\mathrm{i}}-2 z_{\mathrm{i}}$. This might be due to the less pronounced heat-flux amplitude in their study, suggesting that the effect of varying patch size on entrainment interact with the effect of varying heat-flux amplitude.

Moreover, we showed that the impact of surface heterogeneity on entrainment vanishes already for moderate wind speeds when the flow is aligned perpendicular to the border between the differentially-heated patches, consistent with Raasch and Harbusch (2001) and O11. In contrast, if the flow is directed parallel to the border between the differentiallyheated patches, the impact of surface heterogeneity on entrainment persists even for larger wind speeds.

In order to improve our understanding of the effect of surface heterogeneity on entrainment, we developed a new approach by means of the local flux divergence, which allows to infer local information on entrainment and to separate entrainment from encroachment. Compared to the previous studies by HA08 and O11, who inferred local information on entrainment 
from local values of $z_{\mathrm{i}}$, this new approach is more appropriate for studying entrainment over heterogeneous terrain since local values of $z_{\mathrm{i}}$ are determined by encroachment and by the local structure of the interfacial layer rather than by entrainment. Our analysis revealed a maximum of the local entrainment rate over the centre of the warm patch. Due to the existing $\mathrm{SC}$, thermal energy is accumulated over the centre of the warm patch, which allows the formation of strong thermal convection that penetrate rigorously into the capping inversion. Moreover, we also found a maximum of the entrainment rate over the cold patch, which is, in case of large heat-flux amplitudes, even larger compared to the entrainment rate in the homogeneous case. This is in contrast to HA08 and O11 who argued that entrainment is decreased over the cold patch. The large entrainment rate over the cold patch is surprising since the thermal convection, which usually triggers entrainment at the top of the CBL, is completely suppressed by the SC. Our analyses suggest that the large entrainment over the cold patch is mainly due to the horizontal flow convergence induced by the SC, which wrinkles the interfacial layer and thus triggers entrainment events. We hypothesize that the processes that are increasing entrainment depend on the strength of the SC, even though we could not quantify this properly. Furthermore, a counteracting effect exists that is dominant for small heat-flux amplitudes. Due to the horizontal advection of warm and cold air by the horizontal branches of the SC the upper and lower part of the CBL is stabilized, respectively. In case of small heat-flux amplitude thermal convection can thus be slightly weakened, leading to decreased entrainment rates over the warm and over the cold patch, compared to the homogeneous case. We note that the processes induced by the surface heterogeneity that are increasing or decreasing entrainment are identified by qualitative analyses only and thus are hypothetical. Hence, these processes should be further explored to improve understanding of how entrainment is modified by heterogeneous surface heating.

Further on, it should be noted that over natural landscapes the impact of heterogeneous surface heating on entrainment depends not just simply on the strength of the SC. For example, for the LITFASS-2003 experiment Maronga and Raasch (2013) found decreased entrainment for the cases with the strongest SCs. In this case various heterogeneity scales and amplitudes superimpose each other, so that the balance between the heterogeneity-induced processes that increase or decrease entrainment might be modified, which is further complicated by a mean wind.

The present study confirms and connects findings from previous studies, which range from decreased to increased entrainment. For the investigated heterogeneous cases we showed that entrainment was decreased up to $20 \%$ or increased up to $60 \%$ compared to the homogeneous case. This large impact raises the question to what extent entrainment observations over land and subsequent parametrizations (for an overview see e.g. Träumner et al. 2011) are affected by effects of surface heterogeneity. Furthermore, the modified entrainment of sensible heat indicates also modified entrainment of dry air into the CBL, which is a crucial process affecting e.g. the partitioning of surface sensible and latent heat fluxes or cloud formation (van Heerwaarden et al. 2009; Huang and Margulis 2013). Further research is needed to quantify the physics of entrainment over heterogeneous terrain, in order to consider them either in surface or entrainment parametrizations, to improve the representation of the landatmosphere interaction in larger-scale models.

Acknowledgments This study was supported by the German Research Foundation (DFG) under grant RA 617/21-1 and RA 617/20-1, as well as by the Niedersächsische Technische Hochschule (NTH) under grant BU 2.2.3. All simulations were performed on the SGI Altix ICE at The North-German Supercomputing Alliance (HLRN), Hannover/Berlin. NCL (The NCAR Command Language (Version 6.0.0) [software]. (2011). Boulder, Colorado: UCAR/NCAR/CISL/VETS. doi:10.5065/D6WD3XH5) has been used for data analysis 
and visualization. We appreciate the two anonymous reviewers for their constructive and valuable comments that helped to improve the manuscript.

Open Access This article is distributed under the terms of the Creative Commons Attribution License which permits any use, distribution, and reproduction in any medium, provided the original author(s) and the source are credited.

\section{References}

Angevine WM (1999) Entrainment results including advection and case studies from the Flatland boundary layer experiments. J Geophys Res 104:30947-30963

Angevine WM, White AB, Avery SK (1994) Boundary layer depth and entrainment zone characterization with a boundary-layer profiler. Boundary-Layer Meteorol 68:375-385

Avissar R, Schmidt T (1998) An evaluation of the scale at which ground-surface heat flux patchiness affects the convective boundary layer using large-eddy simulations. J Atmos Sci 55:2666-2689

Bange J, Spiess T, van den Kroonenberg A (2007) Characteristics of the early-morning shallow convective boundary layer from Helipod flights during STINHO-2. Theor Appl Climatol 90:113-126

Beyrich F, Gryning SE (1998) Estimation of the entrainment zone depth in a shallow convective boundary layer from sodar data. J Appl Meteorol 37:255-268

Beyrich F, Mengelkamp H (2006) Evaporation over a heterogeneous land surface: EVA_GRIPS and the LITFASS-2003 experiment: an overview. Boundary-Layer Meteorol 121:5-32

Brooks IM, Fowler AM (2012) An evaluation of boundary-layer depth, inversion and entrainment parameters by large-eddy simulation. Boundary-Layer Meteorol 142:245-263

Canut G, Lothon M, Said F, Lohou F (2010) Observation of entrainment at the interface between monsoon flow and the Saharan Air Layer. Q J R Meteorol Soc 136:34-46

Chen F, Avissar R (1994) The impact of land-surface wetness heterogeneity on mesoscale heat fluxes. J Appl Meteorol 33:1323-1340

Conzemius RJ, Fedorovich E (2006) Dynamics of sheared convective boundary layer entrainment. Part I: methodological background and large-eddy simulations. J Atmos Sci 63:1151-1178

Davis KJ, Lenschow DH, Oncley SP, Kiemle C, Ehret G, Giez A, Mann J (1997) Role of entrainment in surface-atmosphere interactions over the boreal forest. J Geophys Res 102:29219-29230

Davis KJ, Gamage N, Hagelberg CR, Kiemle C, Lenschow DH, Sullivan PP (2000) An objective method for deriving atmospheric structure from airborne lidar observations. J Atmos Ocean Technol 17:1455-1468

Deardorff JW (1980) Stratocumulus-capped mixed layers derived from a three-dimensional model. BoundaryLayer Meteorol 18:495-527

Fedorovich E, Conzemius RJ, Mironov D (2004) Convective entrainment into a shear-free, linearly stratified atmosphere: bulk models reevaluated through large eddy simulations. J Atmos Sci 61:281-295

Fesquet C, Dupont S, Drobinski P, Dubos T, Barthlott C (2009) Impact of terrain heterogeneity on coherent structure properties: numerical approach. Boundary-Layer Meteorol 133:71-92

Grabon JS, Davis KJ, Kiemle C, Ehret G (2009) Airborne lidar observations of the transition zone between the convective boundary layer and free atmosphere during the international H2O Project(IHOP) in 2002. Boundary-Layer Meteorol 134:61-83

Huang HY, Margulis SA (2013) Impact of soil moisture heterogeneity length scale and gradients on daytime coupled land-cloudy boundary layer interactions. Hydrol Process 27:1988-2003

Kang SL, Davis KJ, LeMone M (2007) Observations of the ABL structures over a heterogeneous land surface during IHOP_2002. J Hydrometeorol 8:221-244

Kang SL, Davis KJ (2008) The effects of mesoscale surface heterogeneity on the fair-weather convective atmospheric boundary layer. J Atmos Sci 65:3197-3213

Kang SL, Lenschow DH, Sullivan PP (2012) Effects of mesoscale surface thermal heterogeneity on low-level horizontal wind speeds. Boundary-Layer Meteorol 143:409-432

Kim SW, Park SU, Moeng CH (2003) Entrainment processes in the convective boundary layer with varying wind shear. Boundary-Layer Meteorol 108:221-245

Lenschow DH, Mann J, Kristensen L (1994) How long is long enough when measuring fluxes and other turbulence statistics. J Atmos Ocean Technol 11:661-673

Lenschow DH, Krummel PB, Siems ST (1999) Measuring entrainment, divergence, and vorticity on the mesoscale from aircraft. J Atmos Ocean Technol 16:1384-1400

Letzel MO, Raasch S (2003) Large eddy simulation of thermally induced oscillations in the convective boundary layer. J Atmos Sci 60:2328-2341 
Lilly DK (2002) Entrainment into mixed layers. Part I: sharp-edged and smoothed tops. J Atmos Sci 59:33403352

Lothon M, Lenschow DH, Mayor SD (2009) Doppler lidar measurements of vertical velocity spectra in the convective planetary boundary layer. Boundary-Layer Meteorol 132:205-226

Maronga B, Raasch S (2013) Large-eddy simulations of surface heterogeneity effects on the convective boundary layer during the LITFASS-2003 experiment. Boundary-Layer Meteorol 146:17-44

Otte MJ, Wyngaard JC (2001) Stably stratified interfacial-layer turbulence from large-eddy simulation. J Atmos Sci 58:3424-3442

Ouwersloot HG, Vilà-Guerau de Arellano J, van Heerwaarden CC, Ganzeveld LN, Krol MC, Lelieveld J (2011) On the segregation of chemical species in a clear boundary layer over heterogeneous land surfaces. Atmos Chem Phys 11:10681-10704

Patton EG, Sullivan PP, Moeng CH (2005) The influence of idealized heterogeneity on wet and dry planetary boundary layers coupled to the land surface. J Atmos Sci 62:2078-2097

Pino D, Vilà-Guerau de Arellano J, Duynkerke PG (2003) The contribution of shear to the evolution of a convective boundary layer. J Atmos Sci 60:1913-1926

Raasch S, Etling D (1998) Modeling deep ocean convection: large eddy simulation in comparison with laboratory experiments. J Phys Oceanogr 28:1786-1802

Raasch S, Harbusch G (2001) An analysis of secondary circulations and their effects caused by small-scale surface inhomogeneities using large-eddy simulation. Boundary-Layer Meteorol 101:31-59

Raasch S, Schröter M (2001) PALM - a large-eddy simulation model performing on massively parallel computers. Meteorol Z 10:363-372

Steinfeld G, Raasch S, Markkanen T (2008) Footprints in homogeneously and heterogeneously driven boundary layers derived from a lagrangian stochastic particle model embedded into large-eddy simulation. Boundary-Layer Meteorol 129:225-248

Stull RB (1976) The energetics of entrainment across a density interface. J Atmos Sci 33:1260-1267

Stull RB (1988) An introduction to boundary layer meteorology. Kluwer, Dordrecht 666 pp

Sühring M, Raasch S (2013) Heterogeneity-induced heat-flux patterns in the convective boundary layer: can they be detected from observations and is there a blending height? - A large-eddy simulation study for the LITFASS-2003 experiment. Boundary-Layer Meteorol 148:309-331

Sullivan PP, Patton EG (2011) The effect of mesh resolution on convective boundary layer statistics and structures generated by large-eddy simulation. J Atmos Sci 68:2395-2415

Sullivan PP, Moeng CH, Stevens B, Lenschow DH, Mayor SD (1998) Structure of the entrainment zone capping the convective atmospheric boundary layer. J Atmos Sci 55:3042-3064

Träumner K, Kottmeier C, Corsmeier U, Wieser A (2011) Convective boundary-layer entrainment: short review and progress using doppler lidar. Boundary-Layer Meteorol 141:369-391

van Heerwaarden CC, Vilà-Guerau de Arellano J (2008) Relative humidity as an indicator for cloud formation over heterogeneous land surfaces. J Atmos Sci 65:3263-3277

van Heerwaarden CC, Vilà-Guerau de Arellano J, Moene AF, Holtslag AAM (2009) Interactions between dry-air entrainment, surface evaporation and convective boundary-layer development. Q J R Meteorol Soc 135:1277-1291

van Zanten MC, Duynkerke PG, Cuijpers JWM (1999) Entrainment parameterization in convective boundary layers. J Atmos Sci 56:813-828

Wang C, Tian WS, Parker DJ, Marsham JH, Guo ZH (2011) Properties of a simulated convective boundary layer over inhomogeneous vegetation. Q J R Meteorol Soc 137:99-117

Wicker LJ, Skamarock WC (2002) Time-splitting methods for elastic models using forward time schemes. Mon Weather Rev 130:2088-2097

Williamson JH (1980) Low-storage Runge-Kutta schemes. J Comput Phys 35:48-56 